\title{
$\begin{array}{r}\text { WAGENINGEN } \\ \text { UNIVERSITY \& RESEARCH } \\ \hline\end{array}$
}

Unravelling variation in feeding, social interaction and growth patterns among pigs using an agent-based model

Boumans, I. J. M. M., de Boer, I. J. M., Hofstede, G. J., \& Bokkers, E. A. M.

This is a "Post-Print" accepted manuscript, which has been published in "Physiology and Behavior"

This version is distributed under a non-commercial no derivatives Creative Commons (C)) $(\mathcal{\Theta} \Theta$ (CC-BY-NC-ND) user license, which permits use, distribution, and reproduction in any medium, provided the original work is properly cited and not used for commercial purposes. Further, the restriction applies that if you remix, transform, or build upon the material, you may not distribute the modified material.

Please cite this publication as follows:

Boumans, I. J. M. M., de Boer, I. J. M., Hofstede, G. J., \& Bokkers, E. A. M. (2018). Unravelling variation in feeding, social interaction and growth patterns among pigs using an agent-based model. Physiology and Behavior, 191, 100-115. DOI:

10.1016/j. physbeh.2018.03.030

You can download the published version at:

https://doi.org/10.1016/j.physbeh.2018.03.030 


\section{Unravelling variation in feeding, social interaction and growth patterns 2 among pigs using an agent-based model}

Iris J.M.M. Boumans ${ }^{\mathrm{a}, 1}$, Imke J.M. de Boer ${ }^{\mathrm{a}}$, Gert Jan Hofstede ${ }^{\mathrm{b}}$ and Eddie A.M. Bokkers ${ }^{\mathrm{a}}$

${ }^{a}$ Animal Production Systems group, Wageningen University \& Research, P.O. Box 338, 6700 AH Wageningen, the Netherlands

${ }^{\mathrm{b}}$ Information Technology group, Wageningen University \& Research, P.O. Box 8130, $6700 \mathrm{EW}$ Wageningen, the Netherlands

\section{Abstract}

Domesticated pigs, Sus scrofa, vary considerably in feeding, social interaction and growth patterns. This variation originates partly from genetic variation that affects physiological factors and partly from behavioural strategies (avoid or approach) in competitive food resource situations.

Currently, it is unknown how variation in physiological factors and in behavioural strategies among animals contributes to variation in feeding, social interaction and growth patterns in animals. The aim of this study was to unravel causation of variation in these patterns among pigs. We used an agent-based model to explore the effects of physiological factors and behavioural strategies in pigs on variation in feeding, social interaction and growth patterns. Model results show that variation in feeding, social interaction and growth patterns are caused partly by chance, such as time effects and coincidence of conflicts. Furthermore, results show that seemingly contradictory empirical findings in literature can be explained by variation in pig characteristics (i.e. growth potential, positive feedback, dominance, and coping style). Growth potential mainly affected feeding and growth patterns, whereas positive feedback, dominance and coping style affected feeding patterns, social interaction patterns, as well as growth patterns. Variation in behavioural strategies among pigs can reduce aggression at group level, but also make some pigs more susceptible to social constraints inhibiting them from feeding when they want to, especially low-ranking pigs and pigs with a passive coping style. Variation in feeding patterns, such as feeding rate or meal frequency, can indicate social constraints. Feeding patterns, however, can say something different about social constraints at group versus individual level. A combination of feeding patterns, such as a decreased feed intake, an

\footnotetext{
${ }^{1}$ Corresponding author. Tel. +31 3174838 82; e-mail: iris.boumans@wur.nl
} 
increased feeding rate, and an increased meal frequency might, therefore, be needed to measure social constraints at individual level.

Keywords: growing pigs; feeding behaviour; group dynamics; animal welfare; productivity; simulation.

\section{Introduction}

Behavioural feeding patterns, such as feed intake, meal frequency, meal duration and meal size, vary considerably across domesticated pigs, Sus scrofa [e.g. 1, 2, 3]. Although each animal is assumed to reach a certain level of daily food intake, the strategy to reach this differs among animals $[4,5]$. Scientific literature suggests four main feeding patterns in pigs, based on meal frequency, meal duration, and feeding rate [5]. Pigs with few long meals are described as meal eaters, pigs with many short meals as nibblers, pigs with a low feeding rate as slow eaters and pigs with a high feeding rate as fast eaters.

Variation in feeding patterns among pigs partly origins from genetic variation and, therefore, is associated with breeds [5]. Genetic variation can affect pig characteristics, such as growth capacity or stomach size, which can affect physiological processes underlying feeding behaviour, and consequently body weight $[6,7]$. During the growing period, pigs gradually shift from nibblers and slow eaters to meal and fast eaters [8], which can be explained by change in body weight [6].

Pigs of the same breed with a similar body weight, however, still show variation in feeding patterns. In crossbred Landrace $x$ Large White pigs with similar weight, for example, both meal eaters and nibblers were identified [2]. Boumans et al. [9] argued that this kind of variation might result from competition among pigs for feed resources and related behavioural strategies (avoid or approach behaviour). Pigs that avoid conflicts or lose fights, for example, can have limited access to feed in a competitive environment and, therefore, might shift from a meal and slow eater type to a nibbler and fast eater type. In a previous study, we showed that competition can affect feeding rate, 
whereas behavioural strategies in a feed competitive environment can affect meal patterns, such as meal frequency and duration [9].

Currently, it is unknown how physiological processes and behavioural strategies in pigs contribute to consistent, but varying feeding, social interaction and growth patterns among animals. In empirical studies, researchers have tried to explain variation in these patterns based on dominance order. Dominant pigs approached and displaced other pigs more often at the feeder, whereas subordinate pigs are displaced more often at the feeder and showed more but shorter visits to the feeder than dominant pigs [1]. Feeding patterns were reversed in a study of Leiber-Schotte [10], where subordinate boars had fewer and longer meals than dominant boars. Both dominant and subordinate pigs showed high and low feeding visits and displacement attempts in a study of Nielsen et al. [2]. The relation between dominance rank, feeding patterns and social interaction patterns, thus varies between studies. Furthermore, growth rates over the whole growing period were lower for dominant pigs in the study of Leiber-Schotte [10], whereas they were similar for dominant and subordinate pigs in the study of Hoy et al. [1]. This suggests that dominance is important in behavioural strategies, but not fully explains variation in feeding, social interaction and growth patterns of individuals.

Another pig characteristic that potentially might affect behavioural strategies is coping style. Coping styles are regarded as consistent behavioural and physiological responses of animals to environmental challenges [11]. Two typical behavioural coping styles are observed: an aggressive and (pro)active coping style, and a non-aggressive and passive coping style [e.g. 11, 12, 13]. Although the effect of coping styles on feeding patterns in pigs has hardly been studied, typical behaviour associated with coping styles might explain variation in feeding, social interaction and growth patterns in pigs.

Understanding variation in behavioural consistency and plasticity is an intensively studied topic in many feral animal species [14-16]. It is also relevant for domestic farm animals if we want to better 
understand the capacity of animals to cope with environmental factors and their susceptibility to stressors [17]. The aim of this study was to unravel causation of variation in feeding, social interaction and growth patterns among pigs. We hypothesised that interaction between physiological processes and behavioural strategies of individuals, affected by various pig characteristics, can cause consistent behavioural variation and can explain the contrasting results in empirical studies. Understanding the causation of behaviour contributes to recognising normal behaviour and variation in individual pigs, and can help in understanding implications of certain behaviours, for example, for pig growth and welfare.

Important factors in pig behaviour, such as physiological processes and behavioural strategies, are difficult to measure in pigs, as well as their interactive effect on behaviour. One approach to gain more insight into such factors is agent-based modelling. This modelling method allows to test the effect of variation in, and interaction between, factors on (emergent) behavioural patterns in time [18]. In this study, we used an existing agent-based model (ABM) that was developed in a previous study [9]. This ABM explains how physiological processes and behavioural strategies in pigs interact and affect feeding, social interaction and growth patterns in group-housed pigs. In the current study, we used this model to simulate group-housed pigs with varying pig characteristics in a competitive environment. We specifically addressed the following research questions in this model:

1. What is the effect of individual variation in pig characteristics that affect physiological processes on feeding, social interaction and growth patterns?

2. What is the effect of individual variation in pig characteristics that affect behavioural strategies on these patterns?

3. Can interaction between pig characteristics explain empirically observed variation among pigs?

\section{Material \& Methods}

\subsection{Model description}



developed and validated stepwise in previous studies $[6,7,9]$. The model simulates the emergence of feeding, social interaction and growth patterns of group-housed pigs based on physiological factors (e.g. processing of feed, energy absorption, energy use for maintenance, activity and growth) [6], hormonal factors (i.e. circadian rhythms of melatonin and cortisol) [7] and social factors (e.g. competition and social facilitation) [9]. Due to variation in pig characteristics (e.g. growth potential and coping style) various patterns emerge. The model was developed in Netlogo 5.3 [19]. The pattern-oriented modelling (POM) method was used to develop, calibrate and validate the model. This method helps to identify the essential model structure and important processes, and to systematically analyse the model in multiple patterns at different hierarchical levels $[20,21]$. Additionally, sensitivity analyses were performed to evaluate relations between parameter settings and model results [22, 23]. The model version of Boumans et al. [9] was slightly adapted for the current study. Inclusion of social factors (i.e. competition and social facilitation) and behavioural strategies (i.e. avoid and approach) (which was scenario 4 in the previous model) were set as standard in the current study. Additionally, individual variation in pig characteristics was included for four parameters: Mean body protein deposition, Positive feedback, Dominance value and Compete threshold. The main aspects of the model are described below. For a detailed explanation of model structure and processes, see Boumans et al. [9]. Furthermore, the model and a detailed model description are available on the CoMSES website [24] .

\subsubsection{Design concepts}

The model is based on the concept of motivation for behavioural decision-making: internal and external factors affect motivation that causes a behaviour, in which performance of the behaviour has feedback effects [25]. Internal factors include digestion of feed, metabolism, circadian hormonal rhythms and pig characteristics, which affect feeding motivation via their effect on feeding drive and 
specific behaviour consists of an energy drive and threshold level that are affected by these internal and external factors [26]. Subsequently, motivations for several behaviours are compared, in which the highest motivation causes the behaviour, described as the state-space approach by McFarland and Sibly [27]. Performed behaviours affect the energy use and intake of a pig, its growth; and provide feedback to motivation. Feeding, growth and social interaction patterns emerge due to interaction between the above-mentioned factors in the model.

\subsubsection{Agents, environment, state variables and scales}

The model environment represents ten conventionally group-housed agents (pigs) in a barren pen, comparable to a commercial growing pigs housing system. Objects in the pen, besides agents, are a feeder and a drinker. Water and feed are accessible ad libitum. Light (from 06:00 to 18:00 h) and temperature $\left(22^{\circ} \mathrm{C}\right)$ in the pen are based on values commonly found in empirical studies. The feed represents a commercial diet for growing pigs based on values from NRC [28] on required dietary amino acids, protein and digestible energy.

Agents in the model represent growing pigs with various characteristics, which include sex, age, body weight, dominance rank, coping style, growth capacity and meal type. In the current study, pigs represented females (gilts), that started at the age of 70 days and a body weight of about $28 \mathrm{~kg}$ (based on an initial body protein weight of $4 \mathrm{~kg}$ ). Group-housed pigs have a social hierarchy [29, 30]. The social dominance rank of a pig in the model is represented by a randomly assigned and fixed dominance value (mean: 15, value range between 0 and 30). These values correspond to the dominance values used by Hemelrijk [31]. Pigs with higher dominance values represent dominant pigs compared to pigs with lower values that represent subordinate pigs. Pigs have a fixed compete threshold value (mean: 0.3 , range between 0 and 0.6 ) that represents their coping style. Pigs with higher compete threshold values represent passive pigs compared to pigs with lower values that represent active pigs. Pigs with an active coping style, for example, are more likely to initiate an interaction with another pig in a conflict situation [32,33]. Growth capacity of pigs in the model is 
represented by their daily increase in body weight and is based on their minimum body lipid to body protein ratio $(1: 1)$ and their capacity to deposit body protein. Pigs have a fixed mean body protein deposition value (137 g/day, range between 90 and 180) [28, 34]. Pigs with higher mean body protein deposition values have a higher growth capacity and likely have a higher feed intake per day. Meal type of pigs in the model is represented by positive feedback (mean: 0.25 , range between 0 and 0.5). Positive feedback temporarily increases feeding motivation and stimulates a pig to reinforce feeding behaviour in a next time step, thus to increase meal duration and meal size. The value of 0.25 for this parameter was increased compared to Boumans et al. [9] to allow a better assessment of the individual variation effect. A complete overview of state variables and values in the model can be found in the Appendix A, Table A1

Time steps in the model represent one minute within a day of 1440 minutes. Simulations can be run up to 120 days, which represents a 4 month growing period of pigs.

\subsubsection{Process overview}

During each time step, pigs are evaluated in three submodels: Motivation, Behaviour and Growth.

The submodel Motivation includes the calculation of feeding motivation and other behavioural motivations (exploring, drinking or lying). The other behavioural motivations are included to simulate energy use and are based on a drive and threshold that changes every time step. Feeding motivation is included more in depth and is the result of feeding drive and satiation, based on physiological parameters such as stomach load, (instant and daily) energy absorption and requirement. These physiological parameters are affected by circadian patterns of cortisol and melatonin, which vary during the day and affect the daily energy balance and feeding drive. Additionally, feeding motivation of pigs can increase due to feeding behaviour of a group mate, known as a social facilitation effect [35]. 
The submodel Behaviour includes the performance of a behaviour based on the highest motivation. These behaviours include feeding, exploring, drinking or lying. Feeding behaviour can be blocked or disturbed by other pigs. In case of conflicts, hungry pigs can decide to avoid or approach (attempt to displace) other pigs at the feeder, and feeding pigs can be displaced or resist displacement and continue feeding. In a conflict, pigs choose their response based on their Dominance value, Compete threshold and feeding motivation, in which a social higher rank, an active coping style and more hunger will more likely cause an displacement attempt of a hungry pig. When a feeding motivated pigs occupies a feeder, it determines its feeding rate based on a preferred feeding rate (affected by body weight), palatability of the diet and feeding drive. Social pressure (group size effect) can increase the feeding rate of a pig with $0.5 \mathrm{~g} / \mathrm{min}$ per additional pig in the group, but feeding rate cannot exceed a maximum (physically constrained) feeding rate based on body weight.

The submodel Growth calculates nutrient absorption due to digestion and nutrient use for body maintenance, activity and growth per time step. Body weight of pigs is then recalculated based on their nutrient use and intake and growth capacity. Growth capacity depends on their Mean body protein deposition and the ratio of protein and lipid in the body.

\subsection{Simulation experiments}

We simulated six scenarios to test the effect of individual variation in 4 pig characteristics in the model on feeding, social interaction and growth patterns: all pigs with equal pig characteristics (scenario 1), only 1 pig characteristic varied among pigs (scenarios 2 to 5 ), and all 4 pig characteristics varied between pigs (scenario 6) (Table 1). Pig characteristics were individually varied in four parameters: Mean body protein deposition, Positive feedback, Dominance value and Compete threshold. The first two parameters were chosen to represent variation in physiological factors. The parameter Mean body protein deposition represents growth potential and was chosen to affect variation among pigs in the given level of feed intake that a pig aims to reach daily. The parameter 
Positive feedback was chosen to represent meal frequency and duration as it was known that it had a

200 large impact on these patterns in the model [see results of 6]. This parameter might, for example, reflect the capacity of the stomach for feed intake and stimulate longer or shorter meals. The last two parameters, Dominance value and Compete threshold, were chosen to affect variation in behavioural strategies. Dominance value represented dominance rank and Compete threshold represented coping style. These parameters were selected because they are assumed to have a large impact on variation in behavioural strategies (conflict avoidance and approach) without being related to each other. A pig with a more aggressive coping style is not necessarily the most dominant pig in the group that wins fights $[12,13]$, but coping style may affect displacement (attempts) of pigs at the feeder and therefore affect social interaction patterns.

Scenario dependent, parameter values were equal for all pigs (i.e. the mean value) or varied among individuals. If varied, parameter values were randomly assigned to pigs within a pen. In scenario 1 , the four parameters were set equal for all pigs to test to which extent variation in feeding, social interaction and growth patterns is a result of time and competition, rather than the effect of individual variation in pig characteristics and strategies. In scenario 2, 3, 4 and 5 the effect of variation in one of the four parameters (Mean body protein deposition, Positive feedback, Dominance value and Compete threshold) was tested per scenario. Individual variation depended on randomly assigned parameter values to pigs based on a normal distribution with the standard value as mean and a standard deviation that consisted of a percentage of the mean value. A standard deviation of $10 \%$ for Mean body protein deposition was chosen to fit within the range of empirically observed variation in daily protein deposition rates [e.g. 36]. A standard deviation of $30 \%$ for Positive feedback was chosen to create individual variation in which values can also come close to zero. A standard deviation of $30 \%$ for Dominance value was chosen to correspond to the distribution in Hemelrijk [31]. A standard deviation of $30 \%$ for Compete threshold was also chosen to create a range in which values can come close to zero. To prevent a negative value, parameter values of Positive feedback, 

described in Table 1.

Table 1. Scenarios to test the effect of time and individual variation in pig characteristics on feeding, social interaction and growth patterns in groups of 10 pigs.

\begin{tabular}{|c|c|c|c|c|c|c|c|c|}
\hline \multirow[t]{2}{*}{ Scenario } & \multicolumn{4}{|c|}{ Mean value of parameters } & \multicolumn{4}{|c|}{$\begin{array}{l}\text { Percentage of the mean value as } \\
\text { standard deviation }\end{array}$} \\
\hline & 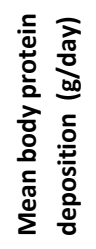 & 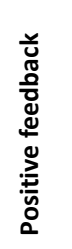 & 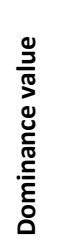 & 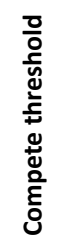 & 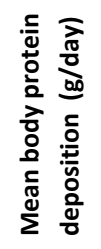 & 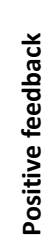 & 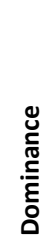 & 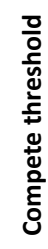 \\
\hline 1. No variation in parameters & 137 & 0.25 & 15 & 0.3 & 0 & 0 & 0 & 0 \\
\hline 2. Growth potential & 137 & 0.25 & 15 & 0.3 & 10 & 0 & 0 & 0 \\
\hline 3. Meal type & 137 & 0.25 & 15 & 0.3 & 0 & 30 & 0 & 0 \\
\hline 4. Dominance & 137 & 0.25 & 15 & 0.3 & 0 & 0 & 30 & 0 \\
\hline 5. Coping style & 137 & 0.25 & 15 & 0.3 & 0 & 0 & 0 & 30 \\
\hline 6. Combined variation & 137 & 0.25 & 15 & 0.3 & 10 & 30 & 30 & 30 \\
\hline
\end{tabular}

230

Sensitivity of the model was tested to the value level of the four chosen parameters (Mean body protein deposition, Positive feedback, Dominance value and Compete threshold) and to the variation among individuals in values for this parameter (Table 2). Scenario 1 was selected to test the effect of parameter values when all values were equal for pigs. The value of each parameter was increased and decreased with $20 \%$ from the standard value in a local sensitivity analysis (thus with change of one parameter value per simulation). Sensitivity of the model to variation among individuals in parameter values was tested in scenario 6 , in which parameter values were different for all pigs representing the scenario closest to a real existing scenario. The standard deviation in the normal distribution when parameter values were assigned to pigs was increased or decreased with $50 \%$ (thus changing the range of variation among individuals). In addition to the four parameters, group size was increased and decreased with 50\% (group sizes 5 and 15) in scenario 6 to test the effect of competition level (i.e. incidence of conflicts). All simulations in this study were run for 14 days, which 

repeated 50 times.

245 Table 2. Sensitivity analysis to test the effect of parameter values and variation of parameter values 246 among individuals on emerged patterns. Changed parameter setting are indicated in bold values.

\begin{tabular}{|c|c|c|c|c|c|c|c|c|c|}
\hline \multirow[t]{2}{*}{ Scenario - parameter - change \% } & \multicolumn{5}{|c|}{ Mean value of parameters } & \multicolumn{4}{|c|}{$\begin{array}{l}\text { Percentage of the mean value } \\
\text { as standard deviation }\end{array}$} \\
\hline & 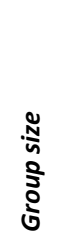 & 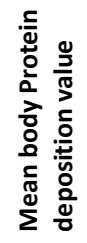 & 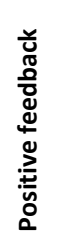 & 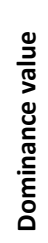 & 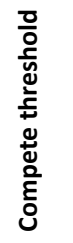 & 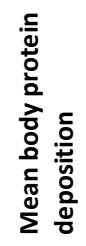 & 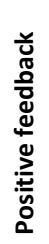 & 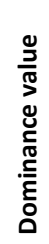 & 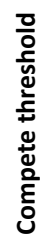 \\
\hline Sc.1 - Mean body protein dep. $-20 \%$ & 10 & 110 & 0.25 & 15 & 0.3 & 0 & 0 & 0 & 0 \\
\hline Sc. 1 - Mean body protein dep. $+20 \%$ & 10 & 164 & 0.25 & 15 & 0.3 & 0 & 0 & 0 & 0 \\
\hline Sc.1 - Positive feedback - $20 \%$ & 10 & 137 & 0.2 & 15 & 0.3 & 0 & 0 & 0 & 0 \\
\hline Sc.1 - Positive feedback + $20 \%$ & 10 & 137 & 0.3 & 15 & 0.3 & 0 & 0 & 0 & 0 \\
\hline Sc.1 - Dominance value - $20 \%$ & 10 & 137 & 0.25 & 12 & 0.3 & 0 & 0 & 0 & 0 \\
\hline Sc. 1 - Dominance value $+20 \%$ & 10 & 137 & 0.25 & 18 & 0.3 & 0 & 0 & 0 & 0 \\
\hline Sc.1 - Compete threshold - $20 \%$ & 10 & 137 & 0.25 & 15 & 0.24 & 0 & 0 & 0 & 0 \\
\hline Sc.1 - Compete threshold + $20 \%$ & 10 & 137 & 0.25 & 15 & 0.36 & 0 & 0 & 0 & 0 \\
\hline Sc. 6 - Mean body protein dep. - $50 \%$ & 10 & 137 & 0.25 & 15 & 0.3 & 5 & 10 & 30 & 30 \\
\hline Sc. $6-$ Mean body protein dep. $+50 \%$ & 10 & 137 & 0.25 & 15 & 0.3 & 15 & 10 & 30 & 30 \\
\hline Sc.6 - Positive feedback - 50\% & 10 & 137 & 0.25 & 15 & 0.3 & 30 & 15 & 30 & 30 \\
\hline Sc.6 - Positive feedback + 50\% & 10 & 137 & 0.25 & 15 & 0.3 & 30 & 45 & 30 & 30 \\
\hline Sc.6- Dominance value - 50\% & 10 & 137 & 0.25 & 15 & 0.3 & 30 & 10 & 15 & 30 \\
\hline Sc. $6-$ Dominance value $+50 \%$ & 10 & 137 & 0.25 & 15 & 0.3 & 30 & 10 & 45 & 30 \\
\hline Sc.6 - Compete threshold - 50\% & 10 & 137 & 0.25 & 15 & 0.3 & 30 & 10 & 30 & 15 \\
\hline Sc. 6 - Compete threshold $+50 \%$ & 10 & 137 & 0.25 & 15 & 0.3 & 30 & 10 & 30 & 45 \\
\hline Sc. 6 - Group size - 50\% & 15 & 137 & 0.25 & 15 & 0.3 & 30 & 10 & 30 & 30 \\
\hline Sc. 6 - Group size $+50 \%$ & 5 & 137 & 0.25 & 15 & 0.3 & 30 & 10 & 30 & 30 \\
\hline
\end{tabular}

Feeding, social interaction and growth patterns on individual and group level were obtained from day 4 to 14 in the model. Feeding patterns were: feed intake (g/day), feeding time (min/day), feeding rate $(\mathrm{g} / \mathrm{min} /$ day), meal frequency (no./day and no./hour), meal duration ( $\mathrm{min} / \mathrm{meal} / \mathrm{day})$, and meal size (g/meal/day). Social interaction patterns were: conflicts (no./day), avoidings (no./day), successful displacements attempts (no./day), unsuccessful displacement attempts (no./day), successful and body weight gain (g/day). 

were analysed using descriptive statistics and general linear models. Corresponding to the experimental period in the study of Nielsen et al. [2], data were averaged over 11 days. Data were analysed at pen level with a general linear model to test the effect of scenarios on feeding, social interaction and growth patterns. When scenarios appeared to be different $(P<0.05)$ a post-hoc pairwise comparison was conducted using Least Squares Means, including an adjustment for multiple comparisons with the Bonferroni test.

In scenarios 2 to 5 , pigs in a pen were ranked and categorised per simulation based on their values for the four parameters Mean body protein deposition, Positive feedback, Dominance value and Compete threshold. The two pigs with the highest value were categorised as high, the two with the lowest value as low, and the remaining pigs were categorised as medium. The average for feeding, social interaction and growth patterns was taken per category and over days. Next, per scenario, high, medium and low categorised pigs were compared for feeding, social interaction and growth patterns using a general linear model. When patterns appeared to be different $(P<0.05)$ a post-hoc pairwise comparison was conducted with a LSD test.

In scenario 6, pigs in a pen were ranked and categorised per simulation based on their averages

272 for feeding, social interaction and growth patterns over 11 days. Two pigs with the lowest average 273 and two pigs with the highest average were selected and respectively categorised based on their meal frequency (meal eater and nibbler), feeding rate (slow and fast eater), conflicts (few conflicts and many conflicts), percentage of displacement attempts to conflicts (avoider and approacher), received displacements received (being avoided and receiver), body weight gain (slow and fast grower). Remaining pigs were categorised as medium. The average for the four parameters Mean body protein deposition, Positive feedback, Dominance value and Compete threshold was taken per category. Next, per pattern, high, medium and low ranking pigs were compared for averages of Mean 
281 linear model. When patterns appeared to be different $(P<0.05)$ a post-hoc pairwise comparison was

282 conducted with a LSD test.

283

284

285

286

287

288

289

290

291

\section{Results}

\subsection{Daily feeding, social interaction and growth patterns at group level}

Mean group patterns of feed intake, feeding time, feeding rate, body weight and body weight

gain were similar in all six scenarios (Table 3). Meal patterns differed between scenarios: meal

frequency was highest in scenarios 1, 2 and 3, and lowest in scenarios 4 and 6 , whereas meal

duration and meal size had opposite results. Also mean social interaction patterns differed between

scenarios: the number of conflicts was lowest in scenario 5 and the number of avoidings was highest

in scenario 6. Displacement attempts (successful and unsuccessful) were highest in scenarios 1, 2 and

3 , and lowest in scenarios 4 and 6.

Table 3. Mean $\pm S D$ of feeding, social interaction and growth patterns at pen level for six scenarios and the $P$-value for differences between scenarios.*

\begin{tabular}{|c|c|c|c|c|c|c|c|}
\hline & $\begin{array}{l}1 . \\
\text { No } \\
\text { variation }\end{array}$ & $\begin{array}{l}2 . \\
\text { Growth } \\
\text { potential }\end{array}$ & $\begin{array}{l}3 . \\
\text { Meal type }\end{array}$ & $\begin{array}{l}4 . \\
\text { Dominance }\end{array}$ & $\begin{array}{l}5 . \\
\text { Coping } \\
\text { style }\end{array}$ & $\begin{array}{l}6 . \\
\text { Combined } \\
\text { variation }\end{array}$ & $\begin{array}{l}P \text { - } \\
\text { value }\end{array}$ \\
\hline \multicolumn{8}{|l|}{ Feeding patterns } \\
\hline Feed intake (g/day) & $1672 \pm 2$ & $1672 \pm 5$ & $1672 \pm 9$ & $1674 \pm 2$ & $1673 \pm 3$ & $1672 \pm 10$ & 0.293 \\
\hline Feeding time (min/day) & $83.7 \pm 0.1$ & $83.7 \pm 0.1$ & $83.6 \pm 0.5$ & $83.8 \pm 0.1$ & $83.7 \pm 0.1$ & $83.7 \pm 0.5$ & 0.221 \\
\hline Feeding rate ( $\mathrm{g} / \mathrm{min} /$ day) & $20.0 \pm 0.0$ & $20.0 \pm 0.1$ & $20.0 \pm 0.0$ & $20.0 \pm 0.0$ & $20.0 \pm 0.0$ & $20.0 \pm 0.1$ & 0.287 \\
\hline Meal frequency (no./day) & $20.7 \pm 0.3^{a}$ & $20.8 \pm 0.4^{\mathrm{a}}$ & $21.1 \pm 0.8^{a}$ & $18.1 \pm 1.0^{\mathrm{b}}$ & $19.4 \pm 1.7^{c}$ & $18.2 \pm 1.8^{\mathrm{b}}$ & $<0.001$ \\
\hline Meal duration (min/meal/day) & $4.2 \pm 0.1^{\mathrm{a}}$ & $4.2 \pm 0.1^{\mathrm{a}}$ & $4.2 \pm 0.2^{\mathrm{a}}$ & $4.8 \pm 0.3^{b}$ & $4.5 \pm 0.4^{c}$ & $4.9 \pm 0.5^{b}$ & $<0.001$ \\
\hline Meal size (g/meal/day) & $85.0 \pm 1.5^{a}$ & $84.4 \pm 1.6^{a}$ & $83.7 \pm 3.5^{\mathrm{a}}$ & $97.1 \pm 5.1^{\mathrm{b}}$ & $90.4 \pm 7.4^{c}$ & $97.8 \pm 8.9^{b}$ & $<0.001$ \\
\hline \multicolumn{8}{|l|}{ Social interaction patterns } \\
\hline Conflicts (no./day) & $130 \pm 3^{\mathrm{ab}}$ & $130 \pm 3^{\mathrm{ab}}$ & $132 \pm 5^{b}$ & $125 \pm 5^{c}$ & $128 \pm 3^{\mathrm{a}}$ & $128 \pm 6^{\text {a }}$ & $<0.001$ \\
\hline Avoidings (no./day) & $102 \pm 2^{\mathrm{a}}$ & $102 \pm 3^{\mathrm{a}}$ & $103 \pm 4^{\mathrm{ab}}$ & $105 \pm 3^{b}$ & $103 \pm 5^{\mathrm{ab}}$ & $108 \pm 6^{c}$ & $<0.001$ \\
\hline \multicolumn{8}{|l|}{ Displacement attempts } \\
\hline Successful (no./day) & $13.8 \pm 0.4^{\mathrm{a}}$ & $13.9 \pm 0.4^{\mathrm{a}}$ & $14.0 \pm 0.5^{\mathrm{a}}$ & $10.4 \pm 1.3^{b}$ & $12.3 \pm 1.9^{c}$ & $10.2 \pm 1.9^{b}$ & $<0.001$ \\
\hline Unsuccessful (no./day) & $13.9 \pm 0.4^{a}$ & $13.9 \pm 0.5^{\mathrm{a}}$ & $14.2 \pm 0.6^{a}$ & $9.8 \pm 1.9^{b}$ & $12.4 \pm 1.9^{c}$ & $9.7 \pm 2.5^{b}$ & $<0.001$ \\
\hline \multicolumn{8}{|l|}{ Growth patterns } \\
\hline Body weight (kg) & $34.9 \pm 0.0$ & $34.8 \pm 0.2$ & $34.8 \pm 0.0$ & $34.9 \pm 0.0$ & $34.9 \pm 0.0$ & $34.8 \pm 0.2$ & 0.324 \\
\hline Body weight gain (g/day) & $834 \pm 1$ & $831 \pm 14$ & $833 \pm 5$ & $834 \pm 0.9$ & $834 \pm 0.9$ & $831 \pm 14$ & 0.073 \\
\hline
\end{tabular}

* The $p$-value of significance levels based on 50 runs per scenario is given for the comparison between scenarios per pattern. Means with different superscripts within a row are significantly different $(P<0.05)$. 
298 categorised as low and high based on their values for the two parameters that are related to physiological factors: Mean body protein deposition and Positive feedback (Table 4). Pigs categorised with a low Mean body protein deposition (Low PD, mean: $119 \mathrm{~g} /$ day) had a significant lower feed intake, higher feeding time, lower feeding rate, lower meal size, lower body weight and lower body weight gain compared to pigs categorised as high body protein deposition potential (High PD, mean: $154 \mathrm{~g} /$ day). Positive feedback affected all patterns, except for successful resists. Pigs categorised with a low Positive feedback value (Low PF, mean: 0.15 ) had a lower feed intake, less feeding time, shorter meal duration, lower meal size, lower body weight, lower body weight gain, and had a higher feeding rate, higher meal frequency, more conflicts, more avoidings and more (successful and unsuccessful)

(High PF, mean: 0.35).

Table 4. Mean $\pm S D$ of feeding, social interaction and growth patterns of pigs low or high in categories of Mean body protein deposition (PD) and Positive feedback (PF) (scenario 2 and 3 ) and the $P$-value for differences between scenarios. *

\begin{tabular}{|c|c|c|c|c|c|c|}
\hline & \multicolumn{2}{|c|}{ Scenario 2. Growth potential } & \multirow[b]{2}{*}{ P-value } & \multicolumn{2}{|c|}{ Scenario 3. Meal type } & \multirow[b]{2}{*}{ P-value } \\
\hline & Low PD & High PD & & Low PF & High PF & \\
\hline \multicolumn{7}{|l|}{ Feeding patterns } \\
\hline Feed intake (g/day) & $1657 \pm 9$ & $1686 \pm 8$ & $<0.001$ & $1641 \pm 15$ & $1703 \pm 12$ & $<0.001$ \\
\hline Feeding time (min/day) & $84.1 \pm 0.3$ & $83.3 \pm 0.3$ & $<0.001$ & $81.8 \pm 0.9$ & $85.5 \pm 0.7$ & $<0.001$ \\
\hline Feeding rate (g/min/day) & $19.7 \pm 0.1$ & $20.3 \pm 0.1$ & $<0.001$ & $20.1 \pm 0.0$ & $20.0 \pm 0.1$ & $<0.001$ \\
\hline Meal frequency (no./day) & $20.9 \pm 0.6$ & $20.7 \pm 0.7$ & 0.369 & $23.1 \pm 1.6$ & $19.5 \pm 0.9$ & $<0.001$ \\
\hline Meal duration (min/meal/day) & $4.2 \pm 0.1$ & $4.2 \pm 0.1$ & 0.830 & $3.7 \pm 0.3$ & $4.6 \pm 0.2$ & $<0.001$ \\
\hline Meal size (g/meal/day) & $83.2 \pm 2.7$ & $85.4 \pm 2.8$ & $<0.001$ & $74.0 \pm 5.8$ & $91.9 \pm 4.7$ & $<0.001$ \\
\hline \multicolumn{7}{|l|}{ Social interaction patterns } \\
\hline Conflicts (no./day) & $132 \pm 7$ & $130 \pm 8$ & 0.092 & $142 \pm 10$ & $122 \pm 6$ & $<0.001$ \\
\hline Avoidings (no./day) & $104 \pm 6$ & $102 \pm 6$ & 0.062 & $112 \pm 8$ & $96 \pm 5$ & $<0.001$ \\
\hline \multicolumn{7}{|l|}{ Displacement attempts } \\
\hline Successful (no./day) & $14.0 \pm 0.6$ & $13.8 \pm 0.7$ & 0.322 & $15.1 \pm 1.0$ & $13.1 \pm 0.7$ & $<0.001$ \\
\hline Unsuccessful (no./day) & $14.0 \pm 1.0$ & $14.1 \pm 1.3$ & 0.590 & $15.2 \pm 1.3$ & $13.2 \pm 1.1$ & $<0.001$ \\
\hline \multicolumn{7}{|l|}{ Receiving displacements } \\
\hline Successful resists (no./day) & $14.0 \pm 0.9$ & $14.0 \pm 0.8$ & 0.782 & $14.1 \pm 1.0$ & $14.3 \pm 1.3$ & 0.792 \\
\hline Displacements (no./day) & $14.0 \pm 0.7$ & $13.9 \pm 0.7$ & 0.637 & $14.1 \pm 1.0$ & $13.7 \pm 0.9$ & 0.023 \\
\hline \multicolumn{7}{|l|}{ Growth patterns } \\
\hline Body weight (kg) & $34.1 \pm 0.3$ & $35.5 \pm 0.3$ & $<0.001$ & $34.7 \pm 0.1$ & $35.0 \pm 0.0$ & $<0.001$ \\
\hline Body weight gain (g/day) & $782 \pm 23$ & $877 \pm 17$ & $<0.001$ & $815 \pm 8$ & $851 \pm 6$ & $<0.001$ \\
\hline
\end{tabular}
pattern and scenario, or if significant, the $p$-value of the pairwise comparison between the high and low category is given. 
316 categorised as low or high based on their values for the two parameters that are related to the

317 behavioural strategies: Dominance value and Compete threshold (Table 5). Pigs categorised as low

318 social rank (Low DOM, mean: 9.0) had a lower feed intake, lower feeding time, shorter meal

319 duration, lower meal size, lower body weight, less successful displacement attempts, lower body

320 weight gain, and had a higher feeding rate, meal frequency, more conflicts, more avoidings, more

321 unsuccessful displacement attempts and more received displacements than pigs categorised as high

322 social rank (High pigs, mean: 20.7). Pigs categorised with a passive coping style (high COMP, mean:

323 0.42) had, comparable to low ranking pigs, a lower feed intake, lower feeding time, higher feeding

324 rate, lower body weight (gain), more conflicts, more avoidings and less successful displacement

325 attempts than pigs with an active coping style (low COMP, mean: 0.18). Passive copers, however, in

326 contrast to low ranking pigs, had fewer, longer and larger meals, and had less unsuccessful

327 displacement attempts and received less (un)successful displacements than active copers.

Table 5. Mean $\pm S D$ of feeding, social interaction and growth patterns of pigs low or high in categories of Dominance value (DOM) and Compete threshold (COMP) (scenario 4 and 5) and the $P$-value for

330 differences between scenarios.*

\begin{tabular}{|c|c|c|c|c|c|c|}
\hline & \multicolumn{3}{|c|}{ Scenario 4. Dominance } & \multicolumn{3}{|c|}{ Scenario 5. Coping style } \\
\hline & Low DOM & High DOM & P-value & Low COMP & High COMP & P-value \\
\hline \multicolumn{7}{|l|}{ Feeding patterns } \\
\hline Feed intake (g/day) & $1600 \pm 27$ & $1714 \pm 9$ & $<0.001$ & $1690 \pm 7$ & $1648 \pm 12$ & $<0.001$ \\
\hline Feeding time ( $\mathrm{min} /$ day) & $79.7 \pm 1.4$ & $86.1 \pm 0.5$ & $<0.001$ & $84.7 \pm 0.4$ & $82.2 \pm 0.7$ & $<0.001$ \\
\hline Feeding rate (g/min/day) & $20.1 \pm 0.0$ & $19.9 \pm 0.0$ & $<0.001$ & $20.0 \pm 0.0$ & $20.1 \pm 0.0$ & $<0.001$ \\
\hline Meal frequency (no./day) & $21.6 \pm 0.9$ & $15.4 \pm 1.0$ & $<0.001$ & $20.4 \pm 1.7$ & $18.1 \pm 2.0$ & $<0.001$ \\
\hline Meal duration (min/meal/day) & $3.8 \pm 0.1$ & $5.7 \pm 0.4$ & $<0.001$ & $4.3 \pm 0.4$ & $4.7 \pm 0.5$ & $<0.001$ \\
\hline Meal size (g/meal/day) & $77.1 \pm 2.7$ & $113.9 \pm 7.3$ & $<0.001$ & $86.7 \pm 7.0$ & $95.5 \pm 9.3$ & $<0.001$ \\
\hline \multicolumn{7}{|l|}{ Social interaction patterns } \\
\hline Conflicts (no./day) & $249 \pm 35$ & $59 \pm 9$ & $<0.001$ & $90 \pm 10$ & $182 \pm 19$ & $<0.001$ \\
\hline Avoidings (no./day) & $228 \pm 41$ & $42 \pm 8$ & $<0.001$ & $61 \pm 8$ & $164 \pm 24$ & $<0.001$ \\
\hline \multicolumn{7}{|l|}{ Displacement attempts } \\
\hline Successful (no./day) & $9.0 \pm 2.8$ & $10.4 \pm 0.8$ & $<0.001$ & $14.7 \pm 1.7$ & $8.9 \pm 3.0$ & $<0.001$ \\
\hline Unsuccessful (no./day) & $11.7 \pm 3.9$ & $7.0 \pm 1.2$ & $<0.001$ & $14.9 \pm 1.9$ & $8.8 \pm 3.0$ & $<0.001$ \\
\hline \multicolumn{7}{|l|}{ Receiving displacements } \\
\hline Successful resists (no./day) & $10.6 \pm 2.3$ & $7.4 \pm 1.9$ & $<0.001$ & $12.8 \pm 2.0$ & $11.8 \pm 2.0$ & 0.058 \\
\hline Displacements (no./day) & $16.4 \pm 0.9$ & $5.6 \pm 1.7$ & $<0.001$ & $12.7 \pm 2.0$ & $11.6 \pm 2.1$ & 0.023 \\
\hline \multicolumn{7}{|l|}{ Growth patterns } \\
\hline Body weight (kg) & $34.6 \pm 0.1$ & $35.0 \pm 0.0$ & $<0.001$ & $34.9 \pm 0.0$ & $34.8 \pm 0.0$ & $<0.001$ \\
\hline Body weight gain (g/day) & $807 \pm 11$ & $851 \pm 3.5$ & $<0.001$ & $841 \pm 3$ & $824 \pm 5$ & $<0.001$ \\
\hline
\end{tabular}

* The $p$-value of significance levels based on 50 runs per scenario is given for the comparison between pig categories per pattern and 
Table 6. Mean $\pm S D$ of parameters values related to pig categories in feeding, social interaction and growth patterns in scenario 6 and the $P$-value for differences between low and high pigs in various

\begin{tabular}{|c|c|c|c|c|c|c|c|c|}
\hline & \multicolumn{4}{|c|}{ Physiological factors } & \multicolumn{4}{|c|}{ Behavioural strategies } \\
\hline & $\begin{array}{l}\text { Mean body } \\
\text { protein } \\
\text { deposition }\end{array}$ & $\begin{array}{l}P \text { - } \\
\text { value }\end{array}$ & $\begin{array}{l}\text { Positive } \\
\text { feedback }\end{array}$ & P-value & $\begin{array}{l}\text { Dominan } \\
\text { ce value }\end{array}$ & P-value & $\begin{array}{l}\text { Compete } \\
\text { threshold }\end{array}$ & P-value \\
\hline \multicolumn{9}{|l|}{ Feeding patterns } \\
\hline Nibbler (22.3 meals/d) & $138 \pm 13$ & \multirow{2}{*}{1.000} & $0.22 \pm 0.08$ & \multirow{2}{*}{$<0.001$} & $10.5 \pm 3.8$ & \multirow{2}{*}{$<0.001$} & $0.25 \pm 0.08$ & \multirow{2}{*}{$<0.001$} \\
\hline Meal eater (14.7 meals/d) & $138 \pm 14$ & & $0.31 \pm 0.06$ & & $18.6 \pm 3.5$ & & $0.34 \pm 0.10$ & \\
\hline Slow eater $(19.8 \mathrm{~g} / \mathrm{min} / \mathrm{d})$ & $126 \pm 12$ & \multirow{2}{*}{$<0.001$} & $0.28 \pm 0.08$ & \multirow{2}{*}{$<0.001$} & $13.6 \pm 4.0$ & \multirow{2}{*}{0.774} & $0.27 \pm 0.08$ & \multirow{2}{*}{$<0.001$} \\
\hline Fast eater $(20.2 \mathrm{~g} / \mathrm{min} / \mathrm{d})$ & $147 \pm 11$ & & $0.22 \pm 0.08$ & & $14.3 \pm 5.0$ & & $0.33 \pm 0.10$ & \\
\hline \multicolumn{9}{|l|}{ Social interaction patterns } \\
\hline Few conflicts ( 56 conflicts/d) & $135 \pm 15$ & \multirow{2}{*}{0.448} & $0.27 \pm 0.07$ & \multirow{2}{*}{0.184} & $19.6 \pm 3.1$ & \multirow{2}{*}{$<0.001$} & $0.24 \pm 0.09$ & \multirow{2}{*}{$<0.001$} \\
\hline Many conflicts (254 conflicts/d) & $138 \pm 13$ & & $0.25 \pm 0.06$ & & $9.4 \pm 3.1$ & & $0.35 \pm 0.10$ & \\
\hline Avoider (approached 7\%) & $138 \pm 14$ & \multirow{2}{*}{0.459} & $0.25 \pm 0.07$ & \multirow{2}{*}{1.000} & $10.5 \pm 3.8$ & \multirow{2}{*}{$<0.001$} & $0.38 \pm 0.09$ & \multirow{2}{*}{$<0.001$} \\
\hline Approacher (approached 36\%) & $135 \pm 14$ & & $0.26 \pm 0.08$ & & $18.3 \pm 3.9$ & & $0.20 \pm 0.07$ & \\
\hline Receiver (27 attempts/d) & $138 \pm 13$ & \multirow{2}{*}{0.680} & $0.26 \pm 0.07$ & \multirow{2}{*}{1.000} & $9.7 \pm 2.9$ & \multirow{2}{*}{$<0.001$} & $0.27 \pm 0.09$ & \multirow{2}{*}{0.003} \\
\hline Being avoided (13 attempts/d) & $136 \pm 14$ & & $0.26 \pm 0.08$ & & $20.1 \pm 3.0$ & & $0.31 \pm 0.09$ & \\
\hline Loser (lost 61\%) & $140 \pm 13$ & \multirow{2}{*}{0.523} & $0.24 \pm 0.07$ & \multirow{2}{*}{0.538} & $9.0 \pm 2.6$ & \multirow{2}{*}{$<0.001$} & $0.29 \pm 0.10$ & \multirow{2}{*}{1.000} \\
\hline Winner (lost 40\%) & $137 \pm 15$ & & $0.26 \pm 0.07$ & & $19.8 \pm 3.0$ & & $0.30 \pm 0.10$ & \\
\hline \multicolumn{9}{|l|}{ Growth patterns } \\
\hline Slow grower $(776 \mathrm{~g} / \mathrm{d})$ & $123 \pm 11$ & \multirow{2}{*}{$<0.001$} & $0.22 \pm 0.07$ & \multirow{2}{*}{$<0.001$} & $12.5 \pm 4.6$ & \multirow{2}{*}{$<0.001$} & $0.31 \pm 0.10$ & \multirow{2}{*}{0.046} \\
\hline Fast grower $(885 \mathrm{~g} / \mathrm{d})$ & $152 \pm 9$ & & $0.29 \pm 0.07$ & & $16.3 \pm 3.9$ & & $0.28 \pm 0.10$ & \\
\hline
\end{tabular}

* The $p$-value of significance levels based on 50 runs per scenario is given for the comparison between all pig categories and if significant the $p$-value between the extreme categories in the pairwise comparison is given.

$339 \quad$ Pig characteristics (based on the four varied parameters) were compared between various

340 categories in feeding, social interaction and growth patterns in scenario 6 (Table 6). Nibblers differed

341 significantly from meal eaters with a lower value for Positive feedback, Dominance value and

342 Compete threshold. Slow eaters had a significantly lower Mean body protein deposition, lower

343 Compete threshold and higher Positive feedback than fast eaters. Pigs with relatively few conflicts

344 had a higher Positive feedback, higher Dominance value and lower Compete threshold value than pigs

345 with relatively many conflicts. Avoiders of conflicts were less dominant and had a higher Compete

346 threshold than approachers of conflicts. Receivers of displacement attempts were less dominant and

347 had a lower Compete threshold than pigs that were being avoided. Losers of interactions had a lower

348 Dominance value than winners of interactions. Slow growers differed from fast growers in all four 


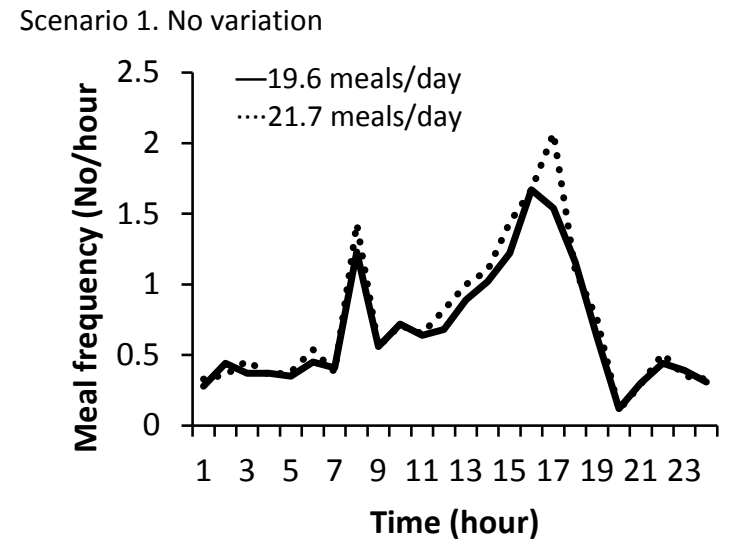

Scenario 3. Meal type

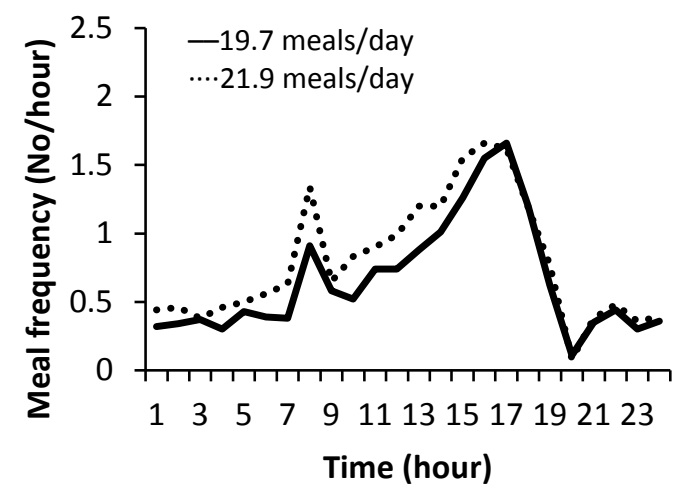

Scenario 5. Coping style

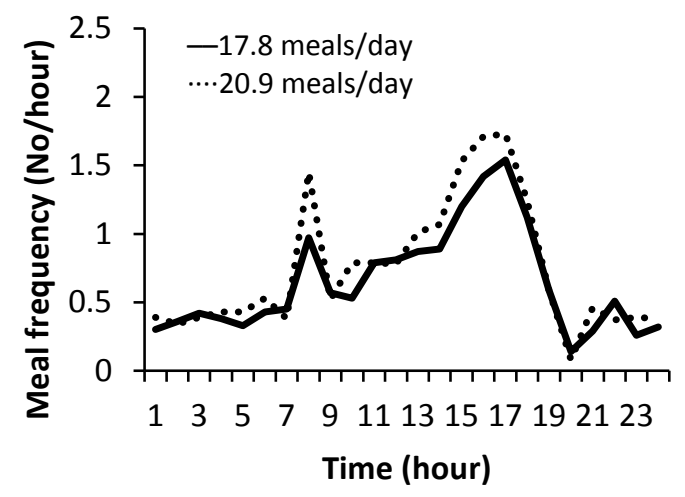

Scenario 2. Growth potential

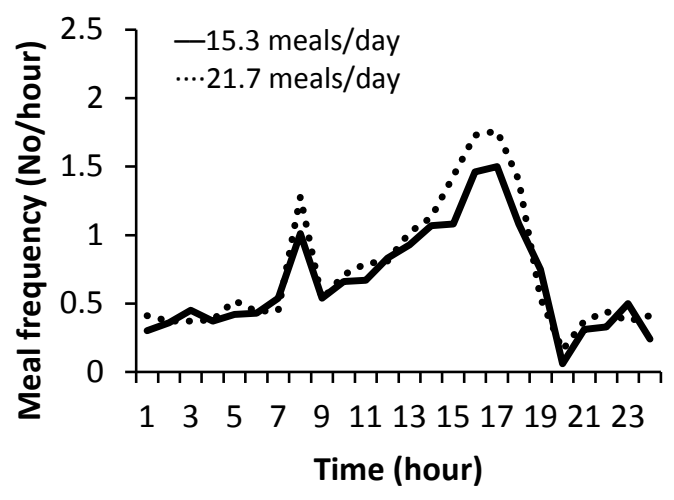

Scenario 4. Dominance

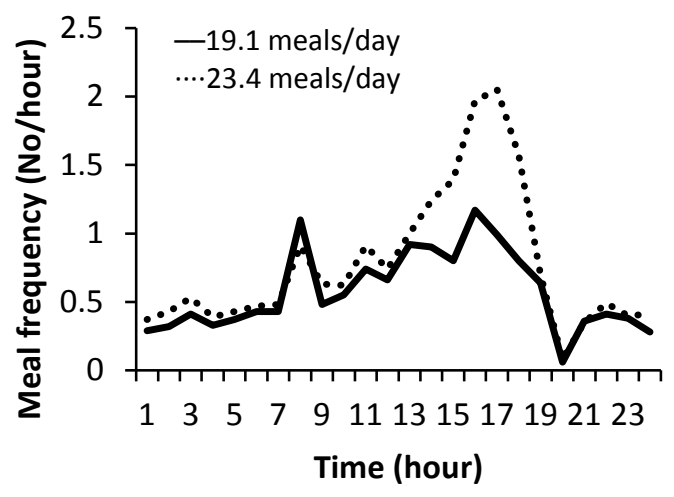

Scenario 6. Combined variation

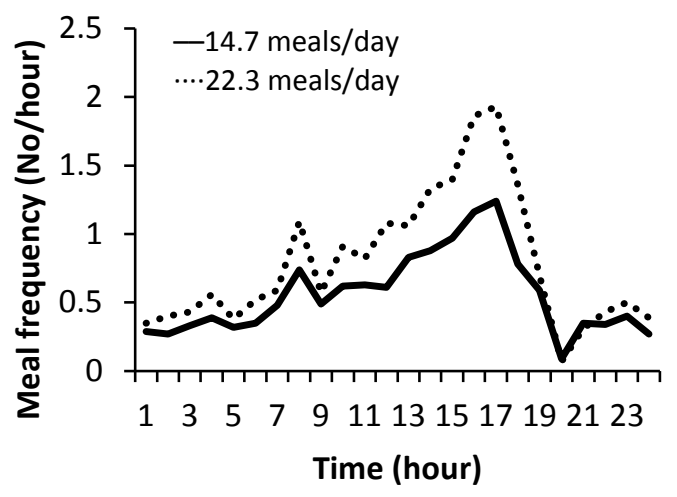

Fig. 1. Hourly mean meal frequency on day 14 for pigs with a daily low (meal eater; - - ) and high meal frequency (Nibbler; …..) per day in the six scenarios. The average values of meals/day for each category (meal eater and nibbler) per scenario are given in the graphs.

The distribution of meal frequency over 24 hours varied between the scenarios for pigs with a low (meal eater) and high meal frequency (nibbler) (Fig. 1). The morning peak between meal eaters 
and nibblers differed most when Positive feedback (Scenario 3), Compete threshold (Scenario 5) and all four parameters (Scenario 6) were varied. The afternoon peak between meal eaters and nibblers differed most when Dominance value was varied (Scenario 4) and all four parameters (Scenario 6) were varied

\subsection{Sensitivity analysis}

Variation by $20 \%$ in the value level of the four parameters (Mean body protein deposition, Positive feedback, Dominance value and Compete threshold) had limited effect $(<20 \%)$ on most model results (Appendix B, Table A2). An exception was Compete threshold, of which an increase resulted in a decrease of displacement patterns (successful and unsuccessful displacement attempts, successful resists and displacements) by $26 \%$ and a decrease increased these patterns by $24 \%$.

\section{Variation among individuals with $50 \%$ in parameter values for the four parameters affected the} mean values and standard deviation with less than $50 \%$ change, whereas variation in group size had an impact on feeding, social interaction and growth patterns of more than $50 \%$ change (Appendix B, Table A3). Increased group size affected mean values and standard deviation of meal frequency and all social interaction patterns, and standard deviation of feeding time and body weight gain.

Decreased group size mainly affected the mean values and standard deviation of social interaction patterns.

\section{Discussion}

The aim of this study was to unravel causation of variation in feeding, social interaction and growth patterns among pigs. We used an ABM to explore the effects of physiological factors and behavioural strategies on behavioural patterns of group-housed pigs. We hypothesised that interaction between physiological factors and behavioural strategies of individuals might affect variation in feeding, social interaction and growth patterns among pigs and can explain the contrasting results in empirical studies. 
Model results showed that variation in feeding, social interaction and growth patterns among pigs is caused partly by chance, from time effects and coincidence of conflicts. In Scenario 1, all pigs were identical for the parameters Mean body protein deposition, Positive feedback, Dominance value and Compete threshold, but they varied in feeding, social interaction and growth patterns. Variation in initial values of motivations for feeding, drinking, exploring and lying at the start of simulations explains these results, but variation can also be partly explained by coincidental conflicts at the feeder.

In real life, pigs can be expected to vary in characteristics that will affect physiological factors and behavioural strategies. Our first research question focussed on the effect of individual variation in pig characteristics that affect physiological processes, tested by individual variation in Mean body protein deposition and Positive feedback. When applied to this model, results showed that variation in Mean body protein deposition, which represented variation in growth capacity, mainly affected feed intake, feeding time, feeding rate, meal size and body weight (gain) of pigs, and partly explained variation in slow and fast eaters and growers (Tables 4 and 6). This is in line with empirical results, in which Landrace and Large White pigs were fast eaters and also had a higher daily feed intake and body weight gain than Pietrain pigs, which were slow eaters [5]. In that same study, Duroc pigs, who similarly to Landrace and Large White pigs had a higher growth potential, appeared slow eaters. These Duroc pigs, however, had the highest meal duration of the four breeds, which was strongly related with feeding rate [5]. This is in line with the model results, in which a higher positive feedback is associated with a higher meal size, a higher daily feed intake, a higher body weight gain and a lower feeding rate (Table 4). This suggests that differences in feeding, social interaction and growth patterns between these breeds can be explained by pig characteristics that affect variation in growth potential and meal duration.

Positive feedback in the model represented a reinforcement effect of feeding that affects meal duration and can be related to, for example, capacity of the stomach and signalling of stomach load. 
Model results showed that variation in Positive feedback affected all feeding and growth patterns in pigs, as well as almost all social interaction patterns (Table 4). A high positive feedback was associated with a higher daily feed intake, eating few but longer meals (meal eater) and fast grower. This is in line with multiple empirical studies, which found an association between increased daily feed intake, large meals, a high feeding rate and daily body weight gain [e.g. 5, 37, 38]. Fernández et al. [5] suggested that pigs with a meal eater and fast eater strategy have a higher productivity. Our study shows how this can be a result of positive feedback that stimulates longer meals.

Our second research question focussed on the effect of individual variation in pig characteristics that affect behavioural strategies of pigs, tested by individual variation in Dominance value and Compete threshold. Model results showed that variation between pigs in Compete threshold affected almost all feeding, social interaction and growth patterns, whereas Dominance value affected all feeding, social interaction and growth patterns (Table 5). Classification in low and high ranking pigs showed that high ranking pigs were mostly meal eaters, whereas low ranking pigs were mostly nibblers. This is in line with empirical results of the study of Hoy et al. [1]. In their study, however, high ranking pigs also had more wins at the feeder than low ranking pigs (respectively 10.3 and 6.9 wins per day at the beginning of the growing period), which slightly differs from our results, in which low ranking pigs had mostly comparable or more wins than high ranking pigs. This might be explained by the assumed hierarchy distribution. Simulated pigs might more easily approach higher ranking pigs than real-life pigs, because of the simplified linear and fixed hierarchy distribution or the effect of probability in decisions to avoid or approach feeding pigs. Especially in the simulated period of the first two weeks, when pigs have a longer daily feeding time and thus more competition, lower ranked pigs are more likely to approach higher ranked pigs.

In the empirical study of Leiber-Schotte [10], where subordinate boars had fewer and longer meals than dominant boars, pigs were fed with electronic feeding stations with protected sides and a rear door that was automatically closed during feeding, protecting feeding pigs from being displaced. 
Although we did not simulate such a feeder, the current results suggest that without displacement possibilities, pigs in group-housing will perform longer meals than usual. This can cause more waiting behaviour for the feeder, in which especially subordinate pigs might have to wait longer, which increases their hunger and motivation for longer meals when they can feed. And since they cannot be displaced from the feeder, it can be expected that once they have reached the feeder, they will perform fewer but longer meals to reach their daily feed intake.

The average number of conflicts and displacement attempts within a group was lowest when variation in Dominance value among pigs was simulated (Table 3). The effect of variation in dominance was expected to reduce aggression, since the dominance order describes the predictable relationship and avoidance order between animals that likely reduces aggression with a more clear dominance order [39]. Also, variation between pigs in Compete threshold, which represented variation in coping style, decreased average displacement attempts within in a group (Table 3 ). The beneficial effect of variation in coping style within group-housed pigs was also shown in an empirical study with homogenous groups of pigs (with either all an active or passive coping style) or heterogeneous groups of pigs having either an active or passive coping style [40]. Agonistic behaviour shortly after mixing was higher in the homogeneous groups consisting of pigs with only active coping styles, than in the other two group types. Furthermore, the mean daily body weight gain was lower in the homogeneous groups consisting of only active copers or only passive copers. This decreased growth is inconsistent with our model results, in which body weight gain was similar between all scenarios. This inconsistency can be explained by the prevalence of health problems in the empirical pigs, which decreased growth especially in the homogeneous groups. body weight gain [e.g. 5, 37, 38], meal eaters had a lower feed intake than nibblers and a comparable body weight gain to nibblers in the study by Nielsen et al. [2]. We hypothesised that this contrast 
research question). Our modelling results show how this contrast can be explained. Results of the empirical study by Nielsen were comparable to our results in scenario 5, with a variation in Compete threshold. In this scenario, meal eaters were pigs with a passive coping style that had a lower feed intake, feeding time, and slightly lower body weight gain than nibblers (for example, see comparison of contrasting patterns between scenario 5 and 6 in Appendix C, Table A4). Since these model patterns were consistent with the empirical results of Nielsen et al. [2], this suggests that meal eaters in the empirical study were pigs with a passive coping style. This is also supported by the hourly patterns of meal frequency, in which a smaller morning peak for meal eater pigs in scenario 5 (Figure 1) is in line with empirical results of Nielsen et al. [2], where pigs had no peak in meal frequency in the morning. Meal eater pigs in that study were suggested to have a disadvantageous feeding strategy. Our results, however, suggest that these pigs might have been pigs with similar feeding strategies (physiological factors), but they might have experienced more social constraints than other group mates due to their passive coping style.

Understanding the causation of individual variation contributes to better understanding of the capacity of animals to cope with environmental factors and their susceptibility to stressors. Feeding patterns in pigs have been found to be consistent over time and flexible when exposed to social competitive situations, however, with variation in coping ability among individuals [41]. Our results show how pig characteristics that affect physiological factors and behavioural strategies can affect the ability of pigs to cope with social constraints. Due to dominance rank, for example, pigs can become meal eaters or nibblers, which can affect their feed intake and aggressive interactions during social constraints. Furthermore, this study contributes to understanding certain behavioural patterns and their implications. This can help, for example, to recognise and use behavioural patterns as indicator for animal welfare problems, such as social constraints and aggression among pigs. Daily feed intake, feeding rate and meal patterns have been suggested to indicate social constraints inhibiting pigs within a group from feeding when they want to. Daily feed intake and body weight gain, for example, decreased as group size increased [42]. Pigs that experience social constraints can 
adapt to these constraints by changing their feeding patterns. If pigs are not able to adapt, however, they might have limited access to the feeder and, therefore, show a decrease in feed intake and body weight gain in comparison to group mates that have similar feed intake requirements and growth potential. A low feed intake and body weight gain, however, can also be associated with other factors, such as a low growth potential (Table 4). Therefore, interpretation of daily feed intake at individual level should be done cautiously. The same caution applies to the use of feeding rate as an indicator of social constraints at individual level. Feeding rate increases in larger group sizes and has been suggested to reflect the social constraints within a group [4, 9]. Our results suggest that feeding rate might not be a suitable indicator at individual level, because it is not only affected by social constraints. Results of slow versus fast eaters in scenario 6 showed that fast eaters were mainly pigs with a high growth potential, low positive feedback and passive coping style (Table 6). Although a low positive feedback and passive coping style are indeed associated with a lower feed intake and daily body weight gain (Table 4 and 5), this is in contrast to a higher growth potential, which had the largest impact on variation in feeding rate and is associated with a higher feed intake and body weight gain (Table 4). Thus feeding rate at individual level might also reflect a higher growth potential of pigs and not necessarily indicate social constraints.

A change in daily meal frequency at group level has also been suggested to be related to social constraints in group-housed pigs, in which an increased meal frequency can indicate increased aggression between pigs and a decreased meal frequency can indicate avoidance behaviour [9]. Although daily meal frequency seems to be a good indicator for social constraints at group level, our results suggest that it might not be a suitable indicator at individual level. As shown in Appendix C and discussed above, a low meal frequency (meal eater pattern) at individual level can be associated with either a high or low feed intake. Therefore, interpretation of meal frequency at individual level should also be done cautiously. This suggests that feeding patterns, such as daily feed intake, meal frequency and feeding rate, by itself might not be good indicators at individual level. 
A combination of feeding patterns might be needed to measure social constraints at individual level. Our results suggest that a high growth potential is associated with a high feed intake and high feeding rate, and therefore, a combination of low daily feed intake and high feeding rate might indicate social constraints. Moreover, these patterns in combination with a low meal frequency might indicate social constraints for a passive coper, whereas these patterns in combination with a high meal frequency might indicate social constraints for a low ranking pig. A low ranking pig, however, shows a feeding pattern comparable to a pig with a low positive feedback. A high afternoon peak in hourly meal frequency is associated with low ranking pigs and can help to differentiate between the effect of a social constraint for a low dominant pig or a physiological effect via a low positive feedback.

\section{The purpose of the model used in this study was to gain deeper understanding of processes} underlying feeding and social interaction behaviour of pigs. The model was developed in multiple steps, in which each step included a validation $[6,7,9]$. Therefore, we expect that the model, even though it is a complex model with many variables, gives a reasonably reliable outcome. Empirical datasets with detailed individual behavioural patterns to confirm this are lacking at this stage, which makes it difficult to validate the findings of the current study. Results of this study are anyhow useful to guide better data collection on potential interesting behavioural patterns. This is especially relevant since advanced technology is currently available to automatically monitor feeding behaviour in pigs and to collect large amounts of data on individual level [e.g. 43, 44]. Understanding what data should be collected, and how it could be analysed and interpreted, can be very useful to find behavioural feeding patterns that can be used as indicator for animal health, welfare and productivity.

To conclude, this study increased understanding of the causation of variation in feeding, social interaction and growth patterns among group-housed pigs. Individual variation in pig characteristics (growth potential, meal type, dominance and coping style) affected many patterns. Growth potential 
531 affected most feeding and growth patterns, but had no effect on social interaction patterns. Meal

532 type and coping style both affected all feeding and growth patterns in pigs, as well as most social

533 interaction patterns (except for successful resists). Dominance affected all feeding, social interaction

534 and growth patterns. Contrasting results in empirical studies on feeding and growth patterns in pigs

535 can be explained by variation in pig characteristics that might interact and cause variation between

536 meal eaters and nibblers and between slow and fast eaters. Individual variation in behavioural

537 strategies can reduce aggression at group level, but can also make some animals more susceptible to

538 social constraints, especially low-ranking pigs and pigs with a passive coping style. Variation in

539 feeding patterns can be an indication of social constraints. A combination of feeding patterns, such

540 as a decreased feed intake, an increased feeding rate, and an increased or decreased meal

541 frequency, might be suitable for identifying individuals that experience social constraints.

\section{Acknowledgements}

543 We would like to thank Aart van der Linden for writing code in R to convert model output into

544 files suitable for analysis. We would like to thank Lydia Nieuwe Weme for advice in model analysis.

545 This research was funded by the IP/OP program 'Complex Adaptive Systems' of Wageningen UR. 
547 Table A1. Global and agents-own state variables, default values or ranges with units of measurement 548 and appearance in model versions. ${ }^{1}$

\begin{tabular}{|c|c|c|c|c|}
\hline Variable & Description & $\begin{array}{l}\text { Default/ range } \\
\text { values }^{2}\end{array}$ & Unit & $\begin{array}{l}\text { Model } \\
\text { version }^{3}\end{array}$ \\
\hline \multicolumn{5}{|c|}{ Globals (variables applied to whole simulation environment, including all agents) } \\
\hline \multicolumn{5}{|l|}{ Time } \\
\hline Days & Number of days since start simulation & $1-120$ & Days & $1,2,3,4$ \\
\hline Minutes & Time of the day in minutes (within 24 hour) & $0-1439$ & Minutes & $1,2,3,4$ \\
\hline Housing & & & & $1,2,3,4$ \\
\hline Housing-size-width & Number of grid cells indicating the size of the pen (width) & 10 & Number & $1,2,3,4$ \\
\hline Housing-size-height & Number of grid cells indicating the size of the pen (height) & 6 & Number & $1,2,3,4$ \\
\hline $\mathrm{Nr}$-of-feeders & Number of feeding spaces (location to feed) & 1 & Number & $1,2,3,4$ \\
\hline Feeders & Location(s) to feed & Patch 0,3 & Grid cell & $1,2,3,4$ \\
\hline Drinker & Location to drink & Patch 9,5 & Grid cell & $1,2,3,4$ \\
\hline Start-light-period & Start of the light period during a day & 6 & Hour & $1,2,3,4$ \\
\hline Start-dark-period & Start of the dark period during a day & 18 & Hour & $1,2,3,4$ \\
\hline Temperature & Ambient temperature in the pen & 22 & Celsius & $1,2,3,4$ \\
\hline Pigs & & & & $1,2,3,4$ \\
\hline Nr-of-gilts & Number of gilts (female pigs) in the pen & $0-30$ & Number & $1,2,3,4$ \\
\hline Nr-of-males & Number of male pigs in the pen & $0-30$ & Number & $1,2,3,4$ \\
\hline Nr-of-barrows & Number of barrows (castrated male pigs) in the pen & $0-30$ & Number & $1,2,3,4$ \\
\hline Initial-weight & Initial body weight of pigs at the start of a simulation & 27 & $\mathrm{Kg}$ & $1,2,3,4$ \\
\hline PO & Initial protein weight of a pig at the start of a simulation & 4 & $\mathrm{Kg}$ & $1,2,3,4$ \\
\hline MinLP-ratio & $\begin{array}{l}\text { Minimum ratio of lipid and protein in the body (separately listed in } \\
\text { the model for gilts, males and barrows) }\end{array}$ & 1 & Unitless (0-1) & $1,2,3,4$ \\
\hline Mean-Pd-gilts & $\begin{array}{l}\text { Mean deposition of body protein that affects growth potential of } \\
\text { gilts }\end{array}$ & 137 & g/day & $1,2,3,4$ \\
\hline Mean-Pd-males & $\begin{array}{l}\text { Mean deposition of body protein that affects growth potential of } \\
\text { males }\end{array}$ & 151 & g/day & $1,2,3,4$ \\
\hline Mean-Pd-barrows & $\begin{array}{l}\text { Mean deposition of body protein that affects growth potential of } \\
\text { barrows }\end{array}$ & 133 & g/day & $1,2,3,4$ \\
\hline DR-MEL-night & Melatonin level during darkness & 0.80 & Unitless (0-1) & $1,2,3,4$ \\
\hline DR-MEL-day & Melatonin level during daylight & 0.40 & Unitless (0-1) & $1,2,3,4$ \\
\hline Cortisol-amplitude & Variation in cortisol levels during the day & 0.99 & Unitless (0-1) & $2,3,4$ \\
\hline Fixed-positive-feedbar & $\begin{array}{l}\text { Reinforcement effect to stimulate continuation when feeding is } \\
\text { performed }\end{array}$ & 0.25 & Unitless (0-1) & $1,2,3,4$ \\
\hline Digest-duration & Total time to digest feed in the gut (passage time in small intestines) & 180 & Minutes & $1,2,3,4$ \\
\hline Compete-threshold & Threshold to compete for access to the feeder & 0.3 & Unitless (0-1) & 3,4 \\
\hline FM-effect-interaction & Effect of feeding motivation to compete for access to the feeder & 0.05 & Unitless (0-1) & 3,4 \\
\hline FR-pig-effect & Represents an increase in FR of $0.5 \mathrm{~g} /$ per pig & 0.5 & $\mathrm{~g}$ & 3,4 \\
\hline $\begin{array}{l}\text { Social-facilitation- } \\
\text { increase }\end{array}$ & $\begin{array}{l}\text { A stimulus that temporarily increases feeding motivation of all not } \\
\text { feeding pigs that time step }\end{array}$ & 0.1 & Unitless (0-1) & 3,4 \\
\hline Increase-lying-energy & Motivational energy increase per time step affecting lying behaviour & 0.033 & Unitless (0-1) & $1,2,3,4$ \\
\hline $\begin{array}{l}\text { Increase-exploring- } \\
\text { energy }\end{array}$ & $\begin{array}{l}\text { Motivational energy increase per time step affecting exploring } \\
\text { behaviour }\end{array}$ & $7.0 \mathrm{E}-4$ & Unitless (0-1) & $1,2,3,4$ \\
\hline $\begin{array}{l}\text { Increase-drinking- } \\
\text { energy }\end{array}$ & $\begin{array}{l}\text { Motivational energy increase per time step affecting drinking } \\
\text { behaviour }\end{array}$ & 0.001 & Unitless (0-1) & $1,2,3,4$ \\
\hline Cost-energy-lying & Motivational energy decrease when lying behaviour performed & 0.054 & Unitless (0-1) & $1,2,3,4$ \\
\hline $\begin{array}{l}\text { Cost-energy- } \\
\text { exploration }\end{array}$ & Motivational energy decrease when exploring behaviour performed & 0.265 & Unitless (0-1) & $1,2,3,4$ \\
\hline Cost-energy-drinking & Motivational energy decrease when drinking behaviour performed & 0.28 & Unitless (0-1) & $1,2,3,4$ \\
\hline Hierarchy? & Variation in dominance values between individuals & Random $30 \%$ & Unitless (0-1) & 4 \\
\hline BW-variation & $\begin{array}{l}\text { Variation in growth capacity of body protein (mean-Pd-gilts) } \\
\text { between individuals }\end{array}$ & 0.10 & Unitless (0-1) & 4 \\
\hline $\begin{array}{l}\text { Coping-style- } \\
\text { variation }\end{array}$ & Variation in coping style (compete-threshold) between individuals & 0.30 & Unitless (0-1) & 4 \\
\hline Pos-fb-variation & Variation in meal type (positive-feedback) between individuals & 0.30 & Unitless (0-1) & 4 \\
\hline
\end{tabular}


DE-content-diet

Palatability

Dietary-AA-content

Digestible energy level of the die

Palatability of the diet

Lysine, Methionine, Methionine+ Cystine, Threonine, Tryptophan and Isoleucine)

Dietary-total-protein- Amount of total protein in the diet

content

Apparent-AA-

Apparent amino acid availabilities in the diet (separately listed in the

availabilities

model for Lysine, Methionine, Methionine+ Cystine, Threonine,

Tryptophan and Isoleucine)

Apparent-protein- Apparent protein availability in the diet

availabilities

Balanced-protein-

AA\%bp

Apparent amino acid utilisation for maintenance (separately listed in the model for Lysine, Methionine, Methionine+ Cystine, Threonine, Tryptophan and Isoleucine)

Gross-energy-content Gross energy content of protein in the feed protein

Agents-own (variables that apply to individual pigs)

\section{Pig characteristics}

Breed Sex of pigs (gilts, barrows and males)

Age Age of the pig

Weight

Body weight of the pig

Dominance-value

Value representing a hierarchical dominance rank in the group

Mean-pd-individual

Capacity to deposit body protein

Ranking

Dominance ranking of pigs (low, medium or high)

Coping-style Coping style of pigs in conflict situations (avoid or approach)

Positive-feedback Meal type of pigs based on a reinforcement effect on feeding

Nutritional \& growth characteristics

PW Part of the body weight consisting of protein

LW Part of the body weight consisting of lipid

Daily-cost-EE Daily energy expenditure for maintenance and activity

Cost-EE-day-before Energy expenditure costs the day before

Sum-GC

Growth capacity for that day

Cost-feeding

Energy costs per digested feed

Metabolic \& physiological characteristics

Meal-list

Time-list

List of feed in the stomach, in amount of feed (g) per intake

List of time of feed (/intake) in the stomach (max $180 \mathrm{~min} /$ intake)

Gut-content

Feed in the gut (representing small/large intestines)

Sum-f-digested

Sum of feed digested in the gut that day

Motivational characteristics

Lying-drive

Sum of motivational energy to perform lying behaviour

Exploring-drive

Sum of motivational energy to perform exploring behaviour

Drinking-drive

Sum of motivational energy to perform drinking behaviour

Sum of performed lying behaviours per pig

Lyings

Explorations

Drinkings

Movements

Waitings

Stay-lyings

Stay-standings

Avoiding

Being-avoided

Active-interaction

Succeed-displacing

Fail-displacing

Displaced

Resisted-displacing

Day-feed-intake

Feeding-minutes

Duration-bout
Sum of performed exploration behaviours per pig

Sum of performed drinking behaviours per pig

Sum of performed movement behaviours per pig

Sum of performed waiting behaviours per pig

Sum of performed remain lying behaviours per pig

Sum of performed remain standing behaviours per pig

Sum of performed avoiding behaviours per pig

Sum of being avoided per pig

Sum of interactions per pig

Sum of successful displacing attempts per pig

Sum of failed displacing attempts per pig

Sum of displacements per pig

Sum of resisted displacements per pig

Sum of feed intake of a pig during the day

Sum of feeding time of a pig during the day

Meal duration
14.2

0.7

2-11

132

$\mathrm{kJ} / \mathrm{g}$

Unitless (0-1)

$1,2,3,4$

$\mathrm{g} / \mathrm{kg}$

$1,2,3,4$

$1,2,3,4$

$\mathrm{g} / \mathrm{kg}$

$1,2,3,4$

Unitless (0-1)

$1,2,3,4$

Unitless (0-1)

$1,2,3,4$

1-7

$\%$

$1,2,3,4$

23.6

$\mathrm{kJ} / \mathrm{g}$

$1,2,3,4$

Gilts

70-190

27-140

$0-30$

90-180

Low-high

0-0.6

0-0.5

4-20

4-50

$-7000-9000$

$-2500-9000$

-

$1,2,3,4$

Days

$\mathrm{Kg}$

$1,2,3,4$

1, 2, 3, 4

Number

3, 4

g/day

3,4

Unitless (0-1)

Unitless (0-1)

4

$\mathrm{Kg}$

$\mathrm{Kg}$

kJ

kJ

12000-35000 kJ

0.09

$\mathrm{kJ} / \mathrm{g}$

Number

Number

$\mathrm{Kg}$

g

$1,2,3,4$

$1,2,3,4$

$1,2,3,4$

$1,2,3,4$

$1,2,3,4$

$2,3,4$

$2,3,4$

$2,3,4$

0-1

0-3500

$1,2,3,4$

$1,2,3,4$

0-0.7

Unitless

$1,2,3,4$

$-0.3-0.3$

Unitless

$1,2,3,4$

$-0.3-0.3$

Unitless

$1,2,3,4$

$1,2,3,4$

Number

$1,2,3,4$

Number

$1,2,3,4$

Number

Number

$1,2,3,4$

$1,2,3,4$

Number

3,4

Number

Number

Number

3,4

3,4

Number

Number

Number

Number

Number

Number

1-3500

g/day

$1-100$

Minutes/day

Minutes/meal
3,4

3,4

3,4

3, 4

3,4

3,4

3,4

1, 2, 3, 4

$1,2,3,4$

$1,2,3,4$ 


\begin{tabular}{|c|c|c|c|c|}
\hline Feeding-bouts & Sum of meals of a pig during the day & $1-80$ & Number/day & $1,2,3,4$ \\
\hline Feed-intake-meal & Amount of feed intake per meal & $20-600$ & $\mathrm{~g} / \mathrm{meal}$ & $1,2,3,4$ \\
\hline $\begin{array}{l}\text { Minutes-since-last- } \\
\text { feeding }\end{array}$ & Time since last meal (interval time between meals) & $1-400$ & Minutes & $1,2,3,4$ \\
\hline Total-meal-interval- & Sum of meal interval time during the day & 1-1390 & Minutes & $1,2,3,4$ \\
\hline
\end{tabular}

time

$549 \quad{ }^{1}$ For a detailed explanation of this Table see the ODD related to the model on the website of CoMSES

550 (https://www.comses.net/codebases/5628/releases/1.1.0/)[24]. ${ }^{2}$ Default or range value in model version $4 .{ }^{3}$ The model described in this

551 study builds on previous models. This column indicates in which version variables were included: $1=1^{\text {st }}$ model published [6], $2=2^{\text {nd }}$ model

552 published [7], $3=3^{\text {rd }}$ model published [9], $4=$ model described in the current study and published on the CoMSES website [24].

553 Appendix B. Sensitivity analysis of parameter values

554 Table A2. Mean values \pm SD of feeding, social interaction and growth patterns in the sensitivity

555 analysis in scenario 1 (all individuals similar parameter values).

\begin{tabular}{|c|c|c|c|c|c|c|c|c|c|}
\hline Pattern & 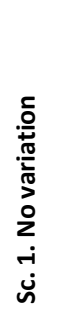 & 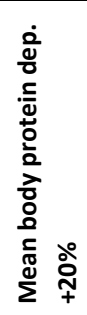 & 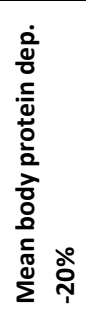 & 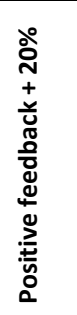 & 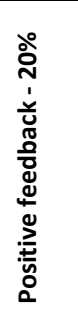 & 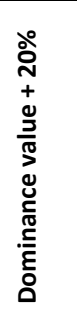 & 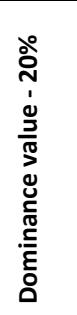 & 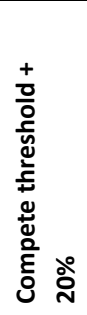 & 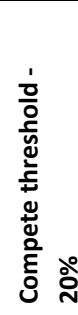 \\
\hline \multicolumn{10}{|l|}{ Feeding patterns } \\
\hline \multirow[t]{2}{*}{ Feed intake (g/day) } & 1672 & 1697 & 1646 & 1690 & 1655 & 1673 & 1673 & 1675 & 1672 \\
\hline & \pm 2 & \pm 2 & \pm 2 & \pm 2 & \pm 2 & \pm 2 & \pm 2 & \pm 2 & \pm 2 \\
\hline \multirow[t]{2}{*}{ Feeding time (min/day) } & 83.7 & 83.3 & 84.1 & 84.7 & 82.7 & 83.7 & 83.7 & 83.8 & 83.6 \\
\hline & \pm 0.1 & \pm 0.1 & \pm 0.1 & \pm 0.1 & \pm 0.1 & \pm 0.1 & \pm 0.1 & \pm 0.1 & \pm 0.1 \\
\hline \multirow[t]{2}{*}{ Feeding rate (g/min/day) } & 20.0 & 20.4 & 19.6 & 20.0 & 20.1 & 20.0 & 20.0 & 20.0 & 20.0 \\
\hline & \pm 0.0 & \pm 0.0 & \pm 0.0 & \pm 0.0 & \pm 0.0 & \pm 0.0 & \pm 0.0 & \pm 0.0 & \pm 0.0 \\
\hline \multirow[t]{2}{*}{ Meal frequency (no./day) } & 20.7 & 19.6 & 21.7 & 19.5 & 22.0 & 20.6 & 20.5 & 17.5 & 23.7 \\
\hline & \pm 0.3 & \pm 0.4 & \pm 0.5 & \pm 0.4 & \pm 0.4 & \pm 0.4 & \pm 0.4 & \pm 0.3 & $\pm 0.6^{\mathrm{s}}$ \\
\hline \multirow[t]{2}{*}{ Meal duration (min/meal/day) } & 4.2 & 4.4 & 4.0 & 4.5 & 3.9 & 4.2 & 4.2 & 4.9 & 3.7 \\
\hline & \pm 0.1 & \pm 0.1 & \pm 0.1 & \pm 0.1 & \pm 0.1 & \pm 0.1 & \pm 0.1 & \pm 0.1 & \pm 0.1 \\
\hline \multirow[t]{2}{*}{ Meal size (g/meal/day) } & 85.0 & 90.3 & 79.6 & 91.0 & 78.7 & 85.2 & 85.4 & 99.2 & 74.8 \\
\hline & \pm 1.5 & \pm 1.7 & \pm 1.7 & \pm 1.8 & \pm 1.4 & \pm 1.5 & \pm 1.8 & \pm 1.6 & \pm 1.6 \\
\hline \multicolumn{10}{|l|}{ Social interaction patterns } \\
\hline \multirow[t]{2}{*}{ Conflicts (no./day) } & 130 & 119 & 141 & 123 & 136 & 129 & 129 & 133 & 126 \\
\hline & \pm 3 & \pm 3 & \pm 3 & \pm 3 & \pm 3 & \pm 3 & \pm 3 & \pm 3 & \pm 3 \\
\hline \multirow[t]{2}{*}{ Avoidings (No./day) } & 102 & 93 & 111 & 97 & 108 & 102 & 102 & 112 & 92 \\
\hline & \pm 2 & \pm 2 & \pm 2 & \pm 2 & \pm 2 & \pm 2 & \pm 2 & \pm 2 & \pm 2 \\
\hline \multicolumn{10}{|l|}{ Displacement attempts } \\
\hline \multirow[t]{2}{*}{ Successful (no./day) } & 13.8 & 12.6 & 15.0 & 13.1 & 14.4 & 13.7 & 13.7 & $10.2^{\mathrm{m}}$ & $17.1^{\mathrm{m}}$ \\
\hline & \pm 0.4 & \pm 0.4 & \pm 0.5 & \pm 0.5 & \pm 0.4 & \pm 0.4 & \pm 0.5 & \pm 0.3 & $\pm 0.6^{\mathrm{s}}$ \\
\hline \multirow[t]{2}{*}{ Unsuccessful (no./day) } & 13.9 & 12.7 & 15.2 & 13.3 & 14.7 & 13.9 & 13.9 & $10.3^{\mathrm{m}}$ & $17.3^{\mathrm{m}}$ \\
\hline & \pm 0.5 & \pm 0.5 & \pm 0.6 & \pm 0.4 & \pm 0.4 & \pm 0.4 & \pm 0.5 & \pm 0.4 & \pm 0.6 \\
\hline \multicolumn{10}{|l|}{ Growth patterns } \\
\hline \multirow[t]{2}{*}{ Body weight (kg) } & 34.9 & 35.8 & 33.8 & 34.9 & 34.8 & 34.9 & 34.9 & 34.9 & 34.9 \\
\hline & \pm 0.0 & \pm 0.0 & \pm 0.0 & 0.0 & \pm 0.0 & \pm 0.0 & \pm 0.0 & \pm 0.0 & \pm 0.0 \\
\hline \multirow[t]{2}{*}{ Body weight gain (g/day) } & 834 & 902 & 754 & 843 & 824 & 834 & 834 & 835 & 834 \\
\hline & \pm 3 & \pm 1 & \pm 1 & \pm 1 & \pm 1 & \pm 1 & \pm 1 & \pm 1 & \pm 3 \\
\hline
\end{tabular}

${ }^{m}$ More than $20 \%$ change in mean values, ${ }^{\mathrm{s}}$ More than $20 \%$ change in SD 
Table A3. Mean values \pm SD of feeding, social interaction and growth patterns in the sensitivity

\begin{tabular}{|c|c|c|c|c|c|c|c|c|c|c|c|}
\hline & 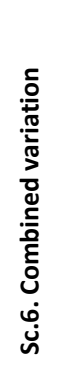 & 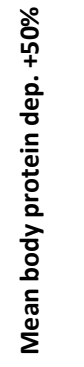 & 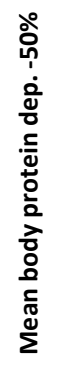 & 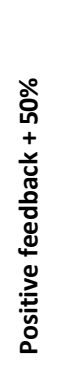 & 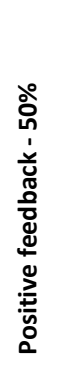 & 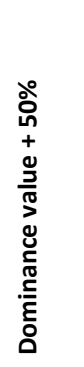 & 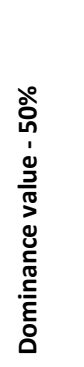 & 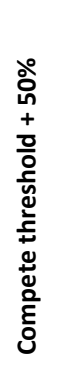 & 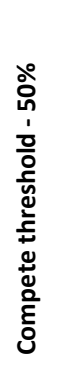 & 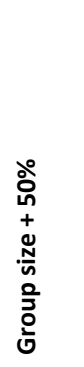 & 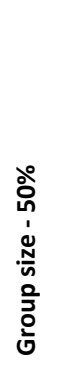 \\
\hline \multicolumn{12}{|l|}{ Feeding patterns } \\
\hline \multirow[t]{2}{*}{ Feed intake (g/day) } & 1672 & 1670 & 1674 & 1670 & 1674 & 1672 & 1673 & 1672 & 1671 & 1560 & 1699 \\
\hline & \pm 10 & \pm 10 & \pm 10 & \pm 11 & \pm 7 & \pm 12 & \pm 11 & \pm 11 & \pm 11 & \pm 8 & $\pm 16^{\mathrm{s}}$ \\
\hline \multirow[t]{2}{*}{ Feeding time (min/day) } & 83.7 & 83.6 & 83.8 & 83.6 & 83.8 & 83.7 & 83.8 & 83.7 & 83.6 & 71.1 & 97.0 \\
\hline & \pm 0.5 & \pm 0.5 & \pm 0.5 & \pm 0.6 & \pm 0.3 & \pm 0.6 & \pm 0.6 & \pm 0.6 & \pm 0.5 & \pm 0.3 & \pm 1.0 \\
\hline \multirow[t]{2}{*}{ Feeding rate $(\mathrm{g} / \mathrm{min} /$ day $)$} & 20.0 & 20.0 & 20.0 & 20.0 & 20.0 & 20.0 & 20.0 & 20.0 & 20.0 & 21.9 & 17.6 \\
\hline & \pm 0.1 & \pm 0.1 & \pm 0.0 & \pm 0.1 & \pm 0.1 & \pm 0.1 & \pm 0.1 & \pm 0.1 & \pm 0.1 & \pm 0.1 & \pm 0.1 \\
\hline \multirow[t]{2}{*}{ Meal frequency (no./day) } & 18.2 & 18.3 & 18.3 & 18.7 & 17.8 & 17.5 & 19.0 & 18.2 & 18.9 & $31.4^{\mathrm{m}}$ & 15.6 \\
\hline & \pm 1.8 & \pm 1.5 & \pm 1.6 & \pm 1.8 & \pm 1.6 & \pm 1.5 & \pm 1.6 & \pm 2.0 & \pm 1.6 & $\pm 4.8^{\mathrm{s}}$ & \pm 1.3 \\
\hline \multirow[t]{2}{*}{ Meal duration ( $\mathrm{min} / \mathrm{meal} /$ day) } & 4.9 & 4.8 & 4.9 & 4.8 & 4.9 & 5.1 & 4.6 & 4.9 & 4.7 & 2.5 & 6.4 \\
\hline & \pm 0.5 & \pm 0.4 & \pm 0.4 & \pm 0.5 & \pm 0.4 & \pm 0.5 & \pm 0.4 & \pm 0.5 & \pm 0.4 & \pm 0.4 & \pm 0.5 \\
\hline \multirow[t]{2}{*}{ Meal size (g/meal/day) } & 97.8 & 96.9 & 97.5 & 96.1 & 99.2 & 102.3 & 93.4 & 97.8 & 93.6 & 55.4 & 112.4 \\
\hline & \pm 8.9 & \pm 7.8 & \pm 8.2 & \pm 9.2 & \pm 8.7 & \pm 9.3 & \pm 8.4 & \pm 10.4 & \pm 7.9 & \pm 9.2 & \pm 9.1 \\
\hline \multicolumn{12}{|l|}{ Social interaction patterns } \\
\hline \multirow[t]{2}{*}{ Conflicts (no./day) } & 128 & 129 & 129 & 131 & 126 & 126 & 129 & 128 & 129 & $305^{m}$ & $32^{\mathrm{m}}$ \\
\hline & \pm 6 & \pm 6 & \pm 6 & \pm 6 & \pm 5 & \pm 6 & \pm 5 & \pm 5 & \pm 6 & $\pm 16^{\mathrm{s}}$ & $\pm 2^{\mathrm{s}}$ \\
\hline \multirow[t]{2}{*}{ Avoidings (No./day) } & 108 & 109 & 108 & 111 & 107 & 110 & 106 & 108 & 108 & $252^{m}$ & $27^{m}$ \\
\hline & \pm 6 & \pm 7 & \pm 6 & \pm 7 & \pm 6 & \pm 5 & \pm 5 & \pm 7 & \pm 5 & \pm 8 & \pm 3 \\
\hline \multicolumn{12}{|l|}{ Displacement attempts } \\
\hline \multirow[t]{2}{*}{ Successful (no./day) } & 10.2 & 10.4 & 10.4 & 10.4 & 9.9 & 9.0 & 11.5 & 10.2 & 11.1 & $26.4^{\mathrm{m}}$ & $2.9^{m}$ \\
\hline & \pm 1.9 & \pm 1.8 & \pm 1.8 & \pm 2.1 & \pm 1.9 & \pm 1.7 & \pm 1.7 & \pm 2.3 & \pm 1.7 & $\pm 5.2^{\mathrm{s}}$ & $\pm 0.6^{\mathrm{s}}$ \\
\hline \multirow[t]{2}{*}{ Unsuccessful (no./day) } & 9.7 & 9.9 & 10.1 & 9.9 & 9.3 & 7.3 & 11.5 & 10.0 & 10.6 & $27.2^{\mathrm{m}}$ & $2.6^{m}$ \\
\hline & \pm 2.5 & \pm 2.5 & \pm 2.1 & \pm 2.5 & \pm 2.4 & \pm 2.6 & \pm 1.8 & \pm 2.6 & \pm 2.1 & $\pm 5.6^{\mathrm{s}}$ & $\pm 0.7^{\mathrm{s}}$ \\
\hline \multicolumn{12}{|l|}{ Growth patterns } \\
\hline \multirow[t]{2}{*}{ Body weight (kg) } & 34.8 & 34.8 & 34.8 & 34.8 & 34.8 & 34.8 & 34.8 & 34.8 & 34.8 & 34.5 & 34.9 \\
\hline & \pm 0.2 & \pm 0.2 & \pm 0.1 & \pm 0.2 & \pm 0.2 & \pm 0.2 & \pm 0.2 & \pm 0.2 & \pm 0.2 & \pm 0.1 & \pm 0.3 \\
\hline \multirow[t]{2}{*}{ Body weight gain (g/day) } & 830 & 829 & 833 & 827 & 831 & 831 & 831 & 831 & 831 & 795 & 838 \\
\hline & \pm 14 & \pm 16 & \pm 8 & \pm 13 & \pm 15 & \pm 13 & \pm 12 & \pm 12 & \pm 15 & \pm 10 & \pm 19 \\
\hline
\end{tabular}


Table A4. Mean $\pm S D$ feeding, social interaction and growth patterns of low and high meal frequency pigs in scenario 5 and 6 . $^{*}$

\begin{tabular}{|c|c|c|c|c|c|c|}
\hline & \multicolumn{3}{|c|}{ Scenario 5 (Compete threshold varied) } & \multicolumn{3}{|c|}{ Scenario 6 (All parameters varied) } \\
\hline & Meal eater & Nibbler & $P$-value & Meal eater & Nibbler & $P$-value \\
\hline \multicolumn{7}{|l|}{ Feeding patterns } \\
\hline Feed intake (g/day) & $1655 \pm 14$ & $1685 \pm 8$ & $<0.001$ & $1719 \pm 31$ & $1625 \pm 37$ & $<0.001$ \\
\hline Feeding time (min/day) & $82.6 \pm 0.8$ & $84.4 \pm 0.5$ & $<0.001$ & $86.3 \pm 1.6$ & $80.9 \pm 2.0$ & $<0.001$ \\
\hline Feeding rate $(\mathrm{g} / \mathrm{min} /$ day $)$ & $20.0 \pm 0.1$ & $20.0 \pm 0.0$ & $<0.001$ & $19.9 \pm 0.2$ & $20.1 \pm 0.2$ & $<0.001$ \\
\hline Meal frequency (no./day) & $17.8 \pm 1.8$ & $20.9 \pm 1.7$ & $<0.001$ & $14.7 \pm 1.4$ & $22.3 \pm 2.6$ & $<0.001$ \\
\hline Meal duration ( $\mathrm{min} / \mathrm{meal} /$ day) & $4.8 \pm 0.4$ & $4.2 \pm 0.3$ & $<0.001$ & $6.0 \pm 0.6$ & $3.8 \pm 0.4$ & $<0.001$ \\
\hline Meal size (g/meal/day) & $97.3 \pm 8.9$ & $84.4 \pm 6.7$ & $<0.001$ & $120.4 \pm 11.8$ & $76.8 \pm 8.8$ & $<0.001$ \\
\hline \multicolumn{7}{|l|}{ Social interaction patterns } \\
\hline Conflicts (no./day) & $167 \pm 25$ & $103 \pm 14$ & $<0.001$ & $79 \pm 33$ & $191 \pm 59$ & $<0.001$ \\
\hline Avoidings (no./day) & $148 \pm 30$ & $74 \pm 14$ & $<0.001$ & $64 \pm 34$ & $164 \pm 63$ & $<0.001$ \\
\hline \multicolumn{7}{|l|}{ Displacement attempts } \\
\hline Successful (no./day) & $9.1 \pm 2.9$ & $14.8 \pm 1.7$ & $<0.001$ & $8.9 \pm 1.7$ & $11.6 \pm 3.9$ & $<0.001$ \\
\hline Unsuccessful (no./day) & $9.4 \pm 3.2$ & $14.6 \pm 1.8$ & $<0.001$ & $6.5 \pm 1.7$ & $14.8 \pm 6.3$ & $<0.001$ \\
\hline \multicolumn{7}{|l|}{ Receiving displacements } \\
\hline Successful resists (no./day) & $11.8 \pm 2.1$ & $12.6 \pm 2.0$ & 0.180 & $8.4 \pm 2.7$ & $10.7 \pm 2.8$ & $<0.001$ \\
\hline Displacements (no./day) & $11.1 \pm 1.9$ & $13.5 \pm 2.1$ & $<0.001$ & $6.3 \pm 2.1$ & $15.1 \pm 3.6$ & $<0.001$ \\
\hline \multicolumn{7}{|l|}{ Growth patterns } \\
\hline Body weight (kg) & $34.8 \pm 0.1$ & $34.9 \pm 0.0$ & $<0.001$ & $35.0 \pm 0.4$ & $34.7 \pm 0.4$ & $<0.001$ \\
\hline Body weight gain (g/day) & $826 \pm 6$ & $839 \pm 3$ & $<0.001$ & $855 \pm 31$ & $813 \pm 27$ & $<0.001$ \\
\hline
\end{tabular}

\section{References}

[1] Hoy, S., Schamun, S., Weirich, C. Investigations on feed intake and social behaviour of fattening pigs fed at an electronic feeding station. Applied Animal Behaviour Science. 2012,139:58-64.

[2] Nielsen, B. L., Lawrence, A. B., Whittemore, C. T. Effect of individual housing on the feeding behaviour of previously group housed growing pigs. Applied Animal Behaviour Science. 1996,47:149-61.

[3] De Haer, L. C. M., Merks, J. W. M. Patterns of daily food intake in growing pigs. Animal Science. 1992,54:95-104.

[4] Nielsen, B. L. On the interpretation of feeding behaviour measures and the use of feeding rate as an indicator of social constraint. Applied Animal Behaviour Science. 1999,63: 79-91.

[5] Fernández, J., Fàbrega, E., Soler, J., Tibau, J., Ruiz, J. L., Puigvert, X., et al. Feeding strategy in grouphoused growing pigs of four different breeds. Applied Animal Behaviour Science. 2011,134:109-20.

[6] Boumans, I. J. M. M., Bokkers, E. A. M., Hofstede, G. J., de Boer, I. J. M. Understanding feeding patterns in growing pigs by modelling growth and motivation. Applied Animal Behaviour Science. 2015, 171:69-80.

[7] Boumans, I. J. M. M., de Boer, I. J. M., Hofstede, G. J., la Fleur, S. E., Bokkers, E. A. M. The importance of hormonal circadian rhythms in daily feeding patterns: An illustration with simulated pigs. Hormones and Behavior. 2017,93: 82-93.

[8] Bigelow, J. A., Houpt, T. R. Feeding and drinking patterns in young pigs. Physiology \& Behavior. 1988,43:99-109.

[9] Boumans, I. J. M. M., de Boer, I. J. M., Hofstede, G. J., Bokkers, E. A. M. How social factors and behavioural strategies affect feeding and social interaction patterns in pigs. (submitted for publication). 2018.

[10] Leiber-Schotte, C. Einfluss der Rangordnung bei Jungebern im Eigenleistungstest auf Futteraufnahme und Futteraufnahmeverhalten unter Berücksichtigung endokrinologischer und immunologischer Parameter. Hannover, Germany: University of Veterinary Medicine; 2009.

[11] Koolhaas, J. M., Korte, S. M., de Boer, S. F., van Der Vegt, B. J., van Reenen, C. G., Hopster, H., et al. Coping styles in animals: current status in behavior and stress-physiology. Neuroscience $\&$ Biobehavioral Reviews. 1999,23: 925-35.

[12] Bolhuis, J. E., Schouten, W. G. P., Schrama, J. W., Wiegant, V. M. Individual coping characteristics, aggressiveness and fighting strategies in pigs. Animal Behaviour. 2005,69:1085-91.

[13] Camerlink, I., Arnott, G., Farish, M., Turner, S. P. Complex contests and the influence of aggressiveness in pigs. Animal Behaviour. 2016,121:71-8. 
[14] Réale, D., Reader, S. M., Sol, D., McDougall, P. T., Dingemanse, N. J. Integrating animal temperament within ecology and evolution. Biological Reviews. 2007,82:291-318.

[15] Sih, A., Bell, A., Johnson, J. C. Behavioral syndromes: An ecological and evolutionary overview. Trends in Ecology and Evolution. 2004,19:372-8.

[16] Wolf, M., Weissing, F. J. An explanatory framework for adaptive personality differences. Philosophical Transactions of the Royal Society B: Biological Sciences. 2010,365:3959-68.

[17] Koolhaas, J. M., van Reenen, C. G. Animal behaviour and well-being symposium: Interaction between coping style/personality, stress, and welfare: Relevance for domestic farm animals1. Journal of Animal Science. 2016,94:2284-96.

[18] Railsback, S. F., Grimm, V. Agent-based and individual-based modeling: a practical introduction. Princeton, New Jersey: Princeton University Press; 2012.

[19] Wilensky, U. Netlogo. Evaston, Illinois: Center for Connected Learning and Computer-Based Modeling, Northwestern University; 1999.

[20] Grimm, V., Railsback, S. F. Pattern-oriented modelling: a 'multi-scope' for predictive systems ecology. Philosophical Transactions of the Royal Society of London B Biological Sciences. 2012,367:298-310.

[21] Grimm, V., Revilla, E., Berger, U., Jeltsch, F., Mooij, W. M., Railsback, S. F., et al. Pattern-oriented modeling of agent-based complex systems: lessons from ecology. Science. 2005,310:987-91.

[22] Ten Broeke, G., van Voorn, G., Ligtenberg, A. Which Sensitivity Analysis Method Should I Use for My Agent-Based Model? J ournal of Artificial Societies and Social Simulation. 2016,19:5.

[23] Lee, J.-S., Filatova, T., Ligmann-Zielinska, A., Hassani-Mahmooei, B., Stonedahl, F., Lorscheid, I., et al. The complexities of agent-based modeling output analysis. Journal of Artificial Societies and Social Simulation. 2015,18:4.

[24] Boumans, I. J. M. M. A model on feeding and social interaction behaviour of pigs. Model version 4. https://www.comses. net/codebases/5628/releases/1.1.0/: CoMSES Computational Model Library; 2018.

[25] J ensen, P., Toates, F. M. Stress as a state of motivational systems. Applied Animal Behaviour Science. 1997,53:145-56.

[26] Hogan, J. A. Energy models of motivation: A reconsideration. Applied Animal Behaviour Science. 1997,53:89-105.

[27] McFarland, D. J., Sibly, R. M. The behavioural final common path. Philos Trans R Soc Lond B Biol Sci. 1975,270:265-93.

[28] NRC. Nutrient requirements of swine. Washington, D.C, USA: National Academies Press; 2012.

[29] Puppe, B., Langbein, J., Bauer, J., Hoy, S. A comparative view on social hierarchy formation at different stages of pig production using sociometric measures. Livestock Science. 2008,113:155-62.

[30] Ewbank, R. Social hierarchy in suckling and fattening pigs: A review. Livestock Production Science. 1976,3:363-72.

[31] Hemelrijk, C. K. Towards the integration of social dominance and spatial structure. Animal Behaviour. 2000,59: 1035-48.

[32] Bolhuis, J. E., Schouten, W. G. P., Schrama, J. W., Wiegant, V. M. Behavioural development of pigs with different coping characteristics in barren and substrate-enriched housing conditions. Applied Animal Behaviour Science. 2005,93:213-28.

[33] Camerlink, I., Turner, S. P., Farish, M., Arnott, G. Aggressiveness as a component of fighting ability in pigs using a game-theoretical framework. Animal Behaviour. 2015,108:183-91.

[34] De Lange, C. F. M. Framework for a simplified model to demonstrate principles of nutrient partitioning for growth in the pig. In: Moughan PJ, Verstegen MWA, Visser-Reyneveld MI, eds. Modelling growth in the pig. Wageningen, the Netherlands: Wageningen Pers; 1995. p. 71-85.

[35] Clayton, D. A. Socially facilitated behavior. The Quarterly Review of Biology. 1978,53:373-92.

[36] Quiniou, N., Dourmad, J.-Y., Noblet, J. Effect of energy intake on the performance of different types of pig from 45 to $100 \mathrm{~kg}$ body weight. 1. Protein and lipid deposition. Animal Science. 1996,63:277-88.

[37] De Haer, L. C. M., Luiting, P., Aarts, H. L. M. Relations among individual (residual) feed intake, growth performance and feed intake pattern of growing pigs in group housing. Livestock Production Science. 1993,36:233-53.

[38] Labroue, F., Gueblez, R., Sellier, P. Genetic parameters of feeding behaviour and performance traits in group-housed Large White and French Landrace growing pigs. Genetics Selection Evolution. 1997,29:451 - 68. [39] Lindberg, A. C. Group Life. In: Keeling LJ, Gonyou HW, eds. Social behavior in farm animals. Wallingford, UK: $C A B$ International; 2001. p. 37-58.

[40] Hessing, M. J. C., Schouten, W. G. P., Wiepkema, P. R., Tielen, M. J. M. Implications of individual behavioural characteristics on performance in pigs. Livestock Production Science. 1994,40:187-96.

[41] Bornett, H. L. I., Morgan, C. A., Lawrence, A. B., Mann, J. The flexibility of feeding patterns in individually housed pigs. Animal Science. 2000,70:457-69.

[42] Hyun, Y., Ellis, M. Effect of group size and feeder type on growth performance and feeding patterns in growing pigs. Journal of Animal Science. 2001,79:803-10.

[43] Maselyne, J., Van Nuffel, A., Briene, P., Vangeyte, J., De Ketelaere, B., Millet, S., et al. Online warning systems for individual fattening pigs based on their feeding pattern. Biosystems Engineering. 2017, in Press.

[44] Matthews, S. G., Miller, A. L., Clapp, J., Plötz, T., Kyriazakis, I. Early detection of health and welfare compromises through automated detection of behavioural changes in pigs. The Veterinary Journal. 2016,217:43-51. 